\title{
Weak compactness and $\sigma$-Asplund generated Banach spaces
}

\author{
by \\ M. Fabian (Praha), V. Montesinos (Valencia) and \\ V. Zizler (Praha)
}

\begin{abstract}
Asplund generated Banach spaces are used to give new characterizations of subspaces of weakly compactly generated spaces and to prove some results on Radon-Nikodým compacta. We show, typically, that in the framework of weakly Lindelöf determined Banach spaces, subspaces of weakly compactly generated spaces are the same as $\sigma$-Asplund generated spaces. For this purpose, we study relationships between quantitative versions of Asplund property, dentability, differentiability, and of weak compactness in Banach spaces. As a consequence, we provide a functional-analytic proof of a result of Arvanitakis: A compact space is Eberlein if (and only if) it is simultaneously Corson and quasi-Radon-Nikodým.
\end{abstract}

1. Definitions and notation. In [9], the results on quantitative versions of the differentiability of norms and of weak compactness were used to give characterizations of several subclasses of weakly Lindelöf determined spaces.

In the present paper, we study a quantitative version of the Asplund property to obtain new characterizations of subspaces of weakly compactly generated spaces. Recall that a Banach space is called Asplund if each separable subspace of it has a separable dual.

Let $(X,\|\cdot\|)$ be a real Banach space with topological dual $X^{*}$. The closed unit balls of $X$ and $X^{*}$ are denoted by $B_{X}$ and $B_{X^{*}}$ respectively. Let $\emptyset \neq M \subset B_{X}$. We define a seminorm $\|\cdot\|_{M}$ on $X^{*}$ by

$$
\left\|x^{*}\right\|_{M}:=\sup \left|\left\langle M, x^{*}\right\rangle\right|:=\sup \left\{\left|\left\langle x, x^{*}\right\rangle\right| ; x \in M\right\}, \quad x^{*} \in X^{*} .
$$

Let $\varepsilon>0$. Given a convex function $f: X \rightarrow \mathbb{R}$, we say that it is $\varepsilon-M$ differentiable at $x \in X$ if

$$
\lim _{t \downarrow 0} \frac{1}{t} \sup \{f(x+t h)+f(x-t h)-2 f(x) ; h \in M\}<\varepsilon .
$$

2000 Mathematics Subject Classification: 46B20, 46B50.

Key words and phrases: $\varepsilon$-Asplund set, $\varepsilon$-weakly compact set, weakly compactly generated Banach space, $\sigma$-Asplund generated space, Radon-Nikodým compact space. 
The norm $\|\cdot\|$ is called $\varepsilon-M$-smooth if it is $\varepsilon-M$-differentiable at each $0 \neq$ $x \in X$. We say that the norm on $X^{*}$, dual to $\|\cdot\|$, and denoted by the same symbol, is $\varepsilon-M-L U R$ if $\lim _{\sup _{n \rightarrow \infty}}\left\|x^{*}-x_{n}^{*}\right\|_{M}<\varepsilon$ whenever $x^{*}, x_{n}^{*} \in$ $B_{X^{*}}, n \in \mathbb{N}$, and $\lim _{n \rightarrow \infty}\left\|x^{*}+x_{n}^{*}\right\|=2$. We say that the dual norm $\|\cdot\|$ on $X^{*}$ has the weak ${ }^{*} \varepsilon-M-$ Kadec property if $\lim \sup _{\tau}\left\|x_{\tau}^{*}-x^{*}\right\|_{M}<\varepsilon$ whenever $x^{*}$ and the net $\left(x_{\tau}^{*}\right)_{\tau \in T}$ lie in the unit sphere $S_{X^{*}}$ of $X^{*}$ and $x_{\tau}^{*} \stackrel{w^{*}}{\rightarrow} x^{*}$. We note that if we have $\varepsilon$ - $M$-smoothness, $\varepsilon$ - $M$-LUR, or weak ${ }^{*} \varepsilon-M$-Kadec property for every $\varepsilon>0$, and $M=B_{X}$, then we get the usual concepts of Fréchet smoothness, LUR, and weak* Kadec property respectively.

Given a non-empty set $A \subset B_{X}$, we say that the closed dual unit ball

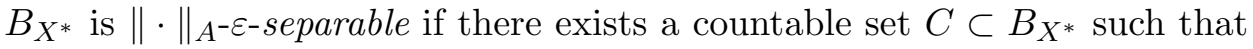
for every $x^{*} \in B_{X^{*}}$ there is $c \in C$ so that $\left\|x^{*}-c\right\|_{A}<\varepsilon$, that is, $B_{X^{*}}$ can be covered by open balls with centers in $C$ and of $\|\cdot\|_{A^{-} \text {radius } \varepsilon \text {. We say }}$ in this case that $C$ is $\|\cdot\|_{A^{-}}$-dense in $B_{X^{*}}$. A subset $M \subset B_{X}$ is said to be $\varepsilon$-Asplund if for every countable subset $\emptyset \neq A \subset M$, the dual unit ball $B_{X^{*}}$

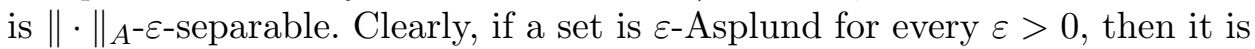
an Asplund set (see [7, Definition 1.4.1]). Note that if $A \subset \varepsilon B_{X}$ then $B_{X^{*}}$

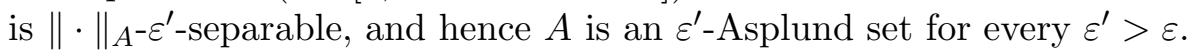

We say that a Banach space $(X,\|\cdot\|)$ is $\sigma$-Asplund generated if for every $\varepsilon>0$ there is a decomposition $B_{X}=\bigcup_{n=1}^{\infty} A_{n}^{\varepsilon}$ where each $A_{n}^{\varepsilon}$ is an $\varepsilon$ Asplund set. We say that the norm $\|\cdot\|$ on $X^{*}$, dual to $\|\cdot\|$, is $\sigma-L U R$ if for every $\varepsilon>0$ there is a decomposition $B_{X}=\bigcup_{n=1}^{\infty} A_{n}^{\varepsilon}$ such that $\|\cdot\|$ is $\varepsilon-A_{n}^{\varepsilon}$-LUR for every $n \in \mathbb{N}$. We say that the norm $\|\cdot\|$ on $X^{*}$, dual to $\|\cdot\|$, has the $\sigma$-weak $k^{*}$ Kadec property if for every $\varepsilon>0$ there is a decomposition $B_{X}=\bigcup_{n=1}^{\infty} A_{n}^{\varepsilon}$ such that $\|\cdot\|$ has the weak* $\varepsilon-A_{n}^{\varepsilon}$-Kadec property for every $n \in \mathbb{N}$. We say that the norm $\|\cdot\|$ on $X$ is $\sigma$-Fréchet smooth if for every $\varepsilon>0$ there is a decomposition $B_{X}=\bigcup_{n=1}^{\infty} A_{n}^{\varepsilon}$ such that the norm $\|\cdot\|$ is $\varepsilon$ - $A_{n}^{\varepsilon}$-differentiable at every $0 \neq x \in X$ for every $n \in \mathbb{N}$.

A Banach space $X$ is called weakly compactly generated $(W C G)$ if it contains a weakly compact set whose linear span is dense in it. $X$ is called weakly Lindelöf determined ( $W L D$ ) if its dual unit ball $B_{X^{*}}$, with the weak* topology, is a Corson compact space. A compact space is called Corson if it is homeomorphic to a subset of $\Sigma(\Gamma):=\left\{u \in \mathbb{R}^{\Gamma} ; \#\{\gamma \in \Gamma ; u(\gamma) \neq 0\} \leq \omega\right\}$, with a suitable set $\Gamma$, endowed with the product topology. A compact space $K$ is called Eberlein if it is homeomorphic to a weakly compact set of $c_{0}(\Gamma)$ in its weak topology, for some set $\Gamma$. We refer to $[4,7,11]$ for the standard notation and results used in this paper.

The paper is organized as follows: Section 2 lists and discusses the main results in this paper. Section 3 studies relationships between the quantitative concepts described above. Most of the statements from this section are then used in Section 4, where the main results are proved. 
2. Characterizations of subspaces of WCG spaces. We start with the following well-known

Theorem 0. Let $X$ be a Banach space. Then $(\mathrm{o}) \Rightarrow(\mathrm{i}) \Leftrightarrow(\mathrm{ii}) \Rightarrow(\mathrm{iii}) \Rightarrow(\mathrm{iv})$ where

(o) $X$ is a WCG and Asplund space.

(i) $X$ admits an equivalent norm whose dual norm is LUR.

(ii) $X$ admits an equivalent norm whose dual norm has the weak ${ }^{*}$ Kadec property.

(iii) $X$ admits an equivalent norm which is Fréchet smooth.

(iv) $X$ is an Asplund space.

Moreover, if $X$ is a WLD space, then all (o)-(iv) are equivalent.

As regards its proof, $(\mathrm{o}) \Rightarrow(\mathrm{i})$ can be found in [4, Theorem VII.1.14]; (i) $\Leftrightarrow($ ii) is from $[26,27]$; for (i) $\Rightarrow($ iii $) \Rightarrow($ iv) see, e.g., [4, Proposition II.1.5 and Theorem II.5.3]; and for the last statement, see, e.g., [7, Theorem 8.3.4].

The following analogue of the above pattern is one of the main results of this paper.

Theorem 1. Let $X$ be a Banach space. Then $(\mathrm{o}) \Rightarrow(\mathrm{i}) \Rightarrow(\mathrm{ii}) \Rightarrow(\mathrm{iv})$ and (o) $\Rightarrow(\mathrm{i}) \Rightarrow(\mathrm{iii}) \Rightarrow(\mathrm{iv})$, where

(o) $X$ is a subspace of a WCG space.

(i) $X$ admits an equivalent norm whose dual norm is $\sigma-L U R$.

(ii) $X$ admits an equivalent norm whose dual norm has the $\sigma$-weak* Kadec property.

(iii) $X$ admits an equivalent norm which is $\sigma$-Fréchet smooth.

(iv) $X$ is a $\sigma$-Asplund generated space.

Moreover, if $X$ is a WLD space, then all (o)-(iv) are equivalent.

A compact space $K$ is called quasi-Radon-Nikodým if there is a lower semicontinuous function $\varrho: K \times K \rightarrow[0,1]$ which separates the points of $K$, and which fragments $K$, i.e., for every $\varepsilon>0$ and every $\emptyset \neq M \subset K$ there is an open set $\Omega \subset K$ so that $M \cap \Omega \neq \emptyset$ and $\sup \{\varrho(k, h) ; k, h \in M \cap \Omega\}<\varepsilon$.

THEOREM 2.

(i) A compact space $K$ is quasi-Radon-Nikodym if and only if the $B a$ nach space $C(K)$ is $\sigma$-Asplund generated.

(ii) A Banach space $X$ is $\sigma$-Asplund generated if and only if $\left(B_{X^{*}}, w^{*}\right)$ is a quasi-Radon-Nikodým compact space.

Here, (i) was proved by Avilés [3, Theorem 20] (see also [8, Theorem 7]), while (ii) follows from [3, Theorem 20], [13, Lemma 4], [8, Theorem 7], and Remark 8 below. 
From Theorems 1 and 2, we get, after a mild effort, the following known

THEOREM 3. For a compact space $K$ the following assertions are equivalent:

(i) $K$ is an Eberlein compact space.

(ii) $K$ is a Corson compact space and $C(K)$ is $\sigma$-Asplund generated.

(iii) $K$ is a Corson and simultaneously quasi-Radon-Nikodým compact space.

The implication (iii) $\Rightarrow(\mathrm{i})$ in Theorem 3 is due to Arvanitakis [2]. In his proof, he used purely topological tools.

Remarks. 1. (i) $\nRightarrow(\mathrm{o})$ in Theorem 1: Let $X$ be the dual $\mathrm{JT}^{*}$ to the James tree space JT [11, pp. 199-201]. Note that JT is separable and that $X^{*}$ is isomorphic to $\mathrm{JT} \oplus \ell_{2}(\Gamma)$. Thus $X^{*}$ admits an equivalent dual LUR norm [4, Theorem VII.2.3(ii)]. If $X$ were a subspace of a WCG space, then JT* would admit an equivalent dual LUR norm [4, Theorem VII.2.3(ii)]. But this would imply that $\mathrm{JT}^{*}$ is separable, a contradiction. Other counterexamples are the spaces $J L_{0}$ or $J L_{2}$ constructed by Johnson and Lindenstrauss (see, e.g. [30]).

2. Concerning the implication (ii) $\Rightarrow$ (i) in Theorem 1 , see the remark at the very end of the paper.

3. (iii) $\nRightarrow\left(\right.$ i) in Theorem 1 : The space $C\left(\left[0, \omega_{1}\right]\right)$ admits an equivalent Fréchet smooth norm [4, Th. 5.4, Ch. VII]. However, it does not satisfy (i). Indeed, it is easy to check that every dual norm which is $\sigma$-LUR is already strictly convex. However, by Talagrand [4, Theorem VII.5.2], $C\left(\left[0, \omega_{1}\right]\right)$ does not admit any equivalent norm whose dual norm would be strictly convex.

4. (iv) $\nRightarrow$ (iii) in Theorem 1: It is easy to check that the $\sigma$-Fréchet smoothness of a norm implies its Gateaux smoothness. Thus any Asplund space which admits no equivalent Gateaux smooth norm provides a counterexample here (see, e.g., [4, Section VII.6]).

5. Every Gateaux smooth norm on a separable Banach space is already $\sigma$-Fréchet smooth (so, in particular, every separable Banach space satisfies (iii) in Theorem 1 and hence it is $\sigma$-Asplund generated, although not always Asplund). Indeed, assume that $(X,\|\cdot\|)$ is a separable Banach space with Gateaux smooth norm, and let $\left\{x_{n} ; n \in \mathbb{N}\right\}$ be a dense subset of $B_{X}$. Put

$$
A_{n}^{\varepsilon}=\left(x_{n}+(\varepsilon / 3) B_{X}\right) \cap B_{X}, \quad n \in \mathbb{N}, \varepsilon>0 .
$$

Then $\bigcup_{n=1}^{\infty} A_{n}^{\varepsilon}=B_{X}$. Moreover, for every $0 \neq x \in X, n \in \mathbb{N}$, and $\varepsilon>0$,

$$
\begin{aligned}
\lim _{t \downarrow 0} \frac{1}{t} \sup \{\| x & \left.+t h\|+\| x-t h\|-2\| x \| ; h \in A_{n}^{\varepsilon}\right\} \\
& \leq \lim _{t \downarrow 0} \frac{1}{t}\left\{\left\|x+t x_{n}\right\|+\left\|x-t x_{n}\right\|-2\|x\|\right\}+\frac{2 \varepsilon}{3}=\frac{2 \varepsilon}{3}<\varepsilon .
\end{aligned}
$$


6. Note that a norm is $\sigma$-Fréchet smooth if it is uniformly Gateaux smooth [10], [9, p. 445]. Thus the $\sigma$-Fréchet smoothness is a common generalization for both the Fréchet smoothness and the uniform Gateaux smoothness. Klee showed in [20] that any separable Banach space $X$ has an equivalent Gateaux smooth norm $\|\cdot\|$ whose dual norm is not strictly convex. Hence this norm on $X$ is not uniformly Gateaux smooth (see [4, Theorem II.6.7]). If, moreover, $X^{*}$ is not separable, then $X$ is not an Asplund space and so the norm $\|\cdot\|$ is not Fréchet smooth; however, according to Remark 5, $\|\cdot\|$ is $\sigma$-Fréchet smooth.

7. Let $X$ be a weakly $\mathcal{K}$-analytic (and so weakly countably determined) Banach space which is not a subspace of a WCG space - one of such spaces, due to Talagrand, can be found, e.g., in [7, Section 4.3]. Let $\|\cdot\|$ be an equivalent Gateaux smooth norm on $X$; it exists according to a result of Mercourakis (see, e.g., [4, Theorem VII.1.16]). This norm is not $\sigma$-Fréchet smooth by Theorem 1 .

8. A Banach space $X$ is called Asplund generated provided that there are an Asplund space $Y$ and a bounded linear mapping $T: Y \rightarrow X$ with $T Y$ dense in $X$. This is equivalent to saying that $X$ contains a linearly dense Asplund subset (see, e.g., [7, Theorem 1.4.4]). In general, this property is not inherited by subspaces: There are examples of subspaces of a WCG Banach space which are not WCG (the first one was given by Rosenthal [28], see also [7, Section 1.6]); use then [7, Theorem 8.3.4]. On the other hand, it is easy to check that subspaces of Asplund generated Banach spaces are $\sigma$-Asplund generated. Indeed, assume that $A$ is an Asplund set generating a Banach space $Z$ and that $X$ is a subspace of $Z$. Then the absolutely convex hull of $A$, say $C$, is also an Asplund set in $Z$. Now, the sets

$$
A_{n}^{\varepsilon}=\left(n C+(\varepsilon / 2) B_{Z}\right) \cap B_{X}, \quad \varepsilon>0, n \in \mathbb{N},
$$

witness that $X$ is $\sigma$-Asplund generated. Also, subspaces of $\sigma$-Asplund generated spaces are $\sigma$-Asplund generated. However, we do not know if $\sigma$-Asplund generated Banach spaces are already subspaces of Asplund generated Banach spaces.

9. A compact space is called Radon-Nikodým if it is homeomorphic to a weak* compact subset of a space that is dual to an Asplund space. A compact space is Radon-Nikodým if and only if it admits a lower semicontinuous metric which fragments it. Trivially, a Radon-Nikodým compact space is quasi-Radon-Nikodým, and it is unknown if the opposite is true. It is easy to show that a continuous image of a quasi-Radon-Nikodým compact space is quasi-Radon-Nikodým. Hence, Theorem 3 implies a result of Stegall that a continuous image of a Radon-Nikodým compact space which is moreover Corson must be Eberlein (see, e.g., [7, Theorem 8.3.6]). Here we 
recall two well-known results. A compact space is Eberlein if and only if it is simultaneously Radon-Nikodým and Corson (see, e.g., [7, Theorem 8.3.5] and references therein). A compact space $K$ is Radon-Nikodym if and only if $C(K)$ is Asplund generated (see, e.g., [7, Theorem 1.5.4]).

10. The implication (iv) $\Rightarrow$ (o) in Theorem 1 for WLD spaces can be deduced once we have Theorem 3 at hand. Indeed, (iv) and [13, Lemma 4] guarantee that $C\left(B_{X^{*}}, w^{*}\right)$ is $\sigma$-Asplund generated. Moreover, if $X$ is WLD then $\left(B_{X^{*}}, w^{*}\right)$ is a Corson compact space. Therefore, by Theorem $3,\left(B_{X^{*}}, w^{*}\right)$ is an Eberlein compact space, and hence $X$ is a subspace of a WCG space (see, e.g. [11, p. 392]).

11. A Banach space $X$ is a subspace of an Asplund generated space if (and only if) $\left(B_{X^{*}}, w^{*}\right)$ is a continuous image of a Radon-Nikodym compact space [7, Theorem 1.5.6]. It is unknown whether every $\sigma$-Asplund generated space is a subspace of an Asplund generated space. Actually, this is equivalent to the question whether every quasi-Radon-Nikodým compact space is a continuous image of a Radon-Nikodým compact space. For more details we refer to [8].

12. Compact spaces $K$ such that $C(K)$ is $\sigma$-Asplund generated are called in [13] countably lower fragmentable.

We postpone the proofs of Theorems 1 and 3 to Section 4, after we prove, in Section 3, results on $\varepsilon$-versions of several qualitative concepts of Banach space theory.

3. $\varepsilon$-concepts. We collect here quantitative versions of known qualitative results on Asplund property, differentiability, dentability, fragmentability, and weak compactness. We shall use them in the proofs of Theorems 1 and 3 . The proofs of such statements are mostly analogous to known proofs of their qualitative counterparts. However, as they are of independent interest, and some more applications of them may be expected in the future, we mostly include their proofs. Sometimes, in the proofs, we are not able to avoid the jump from $\varepsilon$ to $2 \varepsilon$ or $4 \varepsilon$, and we do not have at hand counterexamples demonstrating the necessity of such growth. Actually, we do not care much about this increase since our main objective is the qualitative essence of the quantitative results.

Let $M$ be a non-empty bounded subset of $X$. The $M$-diameter of a set $U \subset X^{*}$ is defined by

$$
M-\operatorname{diam} U:=\sup \left\{\left\|x_{1}^{*}-x_{2}^{*}\right\|_{M} ; x_{1}^{*}, x_{2}^{*} \in U\right\} .
$$

We shall start with a Shmul'yan-like characterization of $\varepsilon$ - $M$-differentiability of a norm; its proof is similar to that of [4, Theorem I.1.4], and hence we omit it. 
Proposition 4. Let $(X,\|\cdot\|)$ be a Banach space, $M \subset B_{X}$ a non-empty subset, $\varepsilon>0$, and $x_{0}$ an element in the unit sphere $S_{X}$ of $X$. Then the following statements are equivalent:

(i) The norm $\|\cdot\|$ is $\varepsilon$-M-differentiable at $x_{0}$.

(ii) For all sequences $\left(x_{n}^{*}\right)$ and $\left(y_{n}^{*}\right)$ in $B_{X^{*}}$ such that $\left\langle x_{0}, x_{n}^{*}\right\rangle \rightarrow 1$ and $\left\langle x_{0}, y_{n}^{*}\right\rangle \rightarrow 1$ as $n \rightarrow \infty$, we have $\left\|x_{n}^{*}-y_{n}^{*}\right\|_{M}<\varepsilon$ for all sufficiently large $n \in \mathbb{N}$.

(iii) The slice $\left\{x^{*} \in B_{X^{*}} ;\left\langle x_{0}, x^{*}\right\rangle>1-\delta\right\}$, with a suitable $\delta>0$, has $M$-diameter less than $\varepsilon$.

Let $\varepsilon>0$ and $\emptyset \neq M \subset B_{X}$ be given. We say that $B_{X^{*}}$ is weak* $\varepsilon-M$-dentable if for every non-empty set $U \subset B_{X^{*}}$ there are $x \in X$ and $\alpha \in \mathbb{R}$ such that the slice $\left\{x^{*} \in U ;\left\langle x, x^{*}\right\rangle>\alpha\right\}$ is non-empty and has $M$-diameter less than $\varepsilon$. We say that $B_{X^{*}}$ is weak $k^{*} \varepsilon$-M-fragmentable if for every non-empty set $U \subset B_{X^{*}}$ there exists a weak* open set $\Omega \subset X^{*}$ such that the intersection $U \cap \Omega$ is non-empty and has $M$-diameter less than $\varepsilon$. Clearly, the weak ${ }^{*} \varepsilon-B_{X}$-dentability (the weak ${ }^{*} \varepsilon-B_{X}$-fragmentability) valid for every $\varepsilon>0$ yields the classical concept of the weak* dentability (resp. fragmentability) by the norm.

The following proposition shows connections between $\varepsilon$-versions of differentiability of functions on the space and the dentability and fragmentability in the dual.

Proposition 5. Let $(X,\|\cdot\|)$ be a Banach space, $M \subset B_{X}$ be a nonempty set, and let $\varepsilon>0$ be given. Then the following assertions are equivalent:

(i) $B_{X^{*}}$ is weak $k^{*} \varepsilon-M$-dentable.

(ii) $B_{X^{*}}$ is weak* $\varepsilon$-M-fragmentable.

(iii) Every convex 1-Lipschitzian function on $X$ is $\varepsilon$-M-differentiable at every point of an open dense subset of $X$.

(iv) Every convex 1-Lipschitzian function on $X$ is $\varepsilon$-M-differentiable at least at one point.

Proof. (i) $\Rightarrow$ (ii) is trivial.

(ii) $\Rightarrow$ (iii). Let $f: X \rightarrow \mathbb{R}$ be a convex 1-Lipschitzian function. Let $U \subset X$ be any non-empty open set. Let $\partial f: X \rightarrow 2^{X^{*}}$ be the MoreauRockafellar subdifferential of $f$ (see $[25$, p. 6$]$ ). $\partial f$ is norm-to-weak* upper semicontinuous [25, Proposition 2.5]. Let $F: X \rightarrow 2^{X^{*}}$ be a minimal normto-weak* upper semicontinuous mapping such that $F(x) \subset \partial f(x)$ for every $x \in X$. Since $f$ is 1-Lipschitzian, $F(U)$ is a subset of $B_{X^{*}}$. Now, (ii) yields a weak* open set $W \subset X^{*}$ such that the set $F(U) \cap W$ is non-empty and has $M$-diameter less than $\varepsilon$. By [7, Lemma 3.1.2], there exists a non-empty open set $\Omega \subset U$ so that $F(\Omega) \subset W$. Then $M$-diam $F(\Omega)<\varepsilon$. We shall show that 
$f$ is $\varepsilon-M$-differentiable at each point of $\Omega$. So fix any $x \in \Omega$. Find $t>0$ so small that $x \pm t M \subset \Omega$. Then for all $h \in M$ we have

$$
\frac{1}{t}(f(x+t h)+f(x-t h)-2 f(x)) \leq\langle h, \xi-\eta\rangle \leq M-\operatorname{diam} F(\Omega)<\varepsilon,
$$

where $\xi \in F(x+t h)$ and $\eta \in F(x-t h)$. We have thus proved that any open set $U \subset X$ contains an open subset $\Omega \subset U$ such that $f$ is $\varepsilon-M$-differentiable at each point of $\Omega$, which is (iii).

(iii) $\Rightarrow$ (iv) is trivial.

(iv) $\Rightarrow$ (i). Fix any non-empty set $U \subset B_{X^{*}}$. Put $f=\sup \langle\cdot, U\rangle$; this is a convex 1-Lipschitzian function on $X$. Find $x_{0} \in X, t>0$, and $\delta>0$ so small that $(1 / t) \sup \left\{f\left(x_{0}+t h\right)+f\left(x_{0}-t h\right)-2 f\left(x_{0}\right) ; h \in M\right\}+3 \delta / t<\varepsilon$. Consider any $x_{1}^{*}, x_{2}^{*}$ in the slice $\left\{x^{*} \in U ;\left\langle x_{0}, x^{*}\right\rangle>f\left(x_{0}\right)-\delta\right\}$. Then for all $h \in M$ we have

$\left\langle x_{0} \pm t h, x_{1}^{*}\right\rangle+\left\langle x_{0} \mp t h, x_{2}^{*}\right\rangle-2 f\left(x_{0}\right) \leq f\left(x_{0} \pm t h\right)+f\left(x_{0} \mp t h\right)-2 f\left(x_{0}\right)<t \varepsilon-3 \delta$, and hence,

$\pm t\left\langle h, x_{1}^{*}-x_{2}^{*}\right\rangle\left\langle t \varepsilon-3 \delta+2 f\left(x_{0}\right)-\left\langle x_{0}, x_{1}^{*}\right\rangle-\left\langle x_{0}, x_{2}^{*}\right\rangle<t \varepsilon-3 \delta+2 \delta=t \varepsilon-\delta\right.$, and so $\left\|x_{1}^{*}-x_{2}^{*}\right\|_{M}<\varepsilon-\delta / t$. Therefore the slice $\left\{x^{*} \in U ;\left\langle x_{0}, x^{*}\right\rangle>\right.$ $\left.f\left(x_{0}\right)-\delta\right\}$ has $M$-diameter not greater than $\varepsilon-\delta / t<\varepsilon$.

REMARK. There exists a direct proof of the implication (ii) $\Rightarrow(\mathrm{i})$. Indeed, it is enough to follow carefully the argument in [24, p. 742].

Let $\varepsilon>0$ and $\emptyset \neq M \subset B_{X}$ be given. We say that the dual ball $B_{X^{*}}$ is $\varepsilon$-M-dentable if for every non-empty set $U \subset B_{X^{*}}$ there are $x^{* *} \in X^{* *}$ and $\alpha \in \mathbb{R}$ such that the slice $\left\{x^{*} \in U ;\left\langle x^{* *}, x^{*}\right\rangle>\alpha\right\}$ is non-empty and has $M$-diameter less than $\varepsilon$. If $B_{X^{*}}$ is $\varepsilon$ - $B_{X^{-}}$-dentable for every $\varepsilon>0$, then we get the usual concept of dentability of $X^{*}$, equivalent, as is well known, to the Radon-Nikodým property of $X^{*}$. Trivially, weak* $\varepsilon-M$-dentability implies $\varepsilon-M$-dentability.

Proposition 6. Let $X$ be a Banach space, $\emptyset \neq M \subset B_{X}, \varepsilon>0$, and assume that the dual unit ball $B_{X^{*}}$ is $\varepsilon-M$-dentable. Then $B_{X^{*}}$ is weak* $2 \varepsilon^{\prime}-M$-dentable for every $\varepsilon^{\prime}>\varepsilon$.

Proof. We follow the proof of [5, Proposition 2]. Fix any $\varepsilon^{\prime}>\varepsilon$. Assume that $B_{X^{*}}$ is not weak ${ }^{*} 2 \varepsilon^{\prime}-M$-fragmentable. Find a set $S \subset B_{X^{*}}$ whose nonempty weak* relatively open subsets have $M$-diameter at least $2 \varepsilon^{\prime}$ each. Take $\varepsilon^{\prime \prime} \in\left(\varepsilon, \varepsilon^{\prime}\right)$. Set $\mathcal{D}=\{\emptyset\} \cup\{0,1\} \cup\{0,1\}^{2} \cup \cdots$. For $d \in \mathcal{D}$ we shall construct weak* relatively open sets $U_{d} \subset S$ and vectors $h_{d} \in M$ such that $U_{d 0} \cup U_{d 1}$ $\subset U_{d}$ and $\inf \left\langle h_{d}, U_{d 0}-U_{d 1}\right\rangle>2 \varepsilon^{\prime \prime}$; here and further we put $d i=\left(d_{1}, \ldots, d_{n}, i\right)$ if $d=\left(d_{1}, \ldots, d_{n}\right) \in \mathcal{D}$ and $i \in\{0,1\}$. Put $U_{\emptyset}=S$. Consider any $d \in \mathcal{D}$ and assume that $U_{d}$ has already been constructed. We know that $\sup \left\langle M, U_{d}-U_{d}\right\rangle$ $>2 \varepsilon^{\prime \prime}$. Find $h_{d} \in M$ and $\xi_{0}, \xi_{1} \in U_{d}$ such that $\left\langle h_{d}, \xi_{0}-\xi_{1}\right\rangle>2 \varepsilon^{\prime \prime}$. Then 
find weak* relatively open sets $U_{d i} \subset U_{d}$ such that $\xi_{i} \in U_{d i}, i=0,1$, and $\inf \left\langle h_{d}, U_{d 0}-U_{d 1}\right\rangle>2 \varepsilon^{\prime \prime}$. This finishes the induction step.

For $d \in \mathcal{D}$ let $K_{d}=\overline{\operatorname{co} U}_{d}{ }^{*}$ denote the weak ${ }^{*}$ closed convex hull of $U_{d}$. We note that $K_{d 0} \cup K_{d 1} \subset K_{d}$, and hence $\frac{1}{2}\left(K_{d 0}+K_{d 1}\right) \subset K_{d}$ for every $d \in \mathcal{D}$. We claim that there exists $t=\left(t_{d} ; d \in \mathcal{D}\right) \in \prod_{d \in \mathcal{D}} K_{d}$ such that $t_{d}=\frac{1}{2}\left(t_{d 0}+t_{d 1}\right)$ for every $d \in \mathcal{D}$; note that the set $\left\{t_{d} ; d \in \mathcal{D}\right\}$ is called a dyadic tree. Clearly, in order to prove the claim, it is enough to show that $\bigcap_{d \in \mathcal{D}} A_{d} \neq \emptyset$, where

$$
A_{d}=\left\{\left(t_{d} ; d \in \mathcal{D}\right) \in \prod_{d \in \mathcal{D}} K_{d} ; t_{d}=\frac{1}{2}\left(t_{d 0}+t_{d 1}\right)\right\}, \quad d \in \mathcal{D} .
$$

Using a compactness argument, it is enough to prove that $\bigcap\left\{A_{d} ; d \in \mathcal{D}\right.$, $|d| \leq n\}$ is non-empty for every $n \in \mathbb{N}$. So fix $n \in \mathbb{N}$. For $d \in \mathcal{D}$ with $|d|>n$ let $t_{d}$ be any element of $K_{d}$. To give the definition for $d \in \mathcal{D}$ with $|d| \leq n$, we use downward induction. Fix any $d \in \mathcal{D}$, with $|d| \leq n$, and assume that we have already defined $t_{d 0} \in K_{d 0}$ and $t_{d 1} \in K_{d 1}$. Put then $t_{d}=\frac{1}{2}\left(t_{d 0}+t_{d 1}\right)$; hence $t_{d} \in K_{d}$. Thus we finally construct $t_{d}$ for every $d \in \mathcal{D}$. It is clear that every $\left(t_{d} ; d \in \mathcal{D}\right)$ from the non-empty set $\bigcap_{n=1}^{\infty}\left\{A_{d} ; d \in \mathcal{D},|d| \leq n\right\}$ satisfies the claim.

Pick some $\left(t_{d} ; d \in \mathcal{D}\right) \in \prod_{d \in \mathcal{D}} K_{d}$. By the assumption, there is a weak open halfspace $V \subset X^{*}$ such that the set $\left\{t_{d} ; d \in \mathcal{D}\right\} \cap V$ is non-empty and has $M$-diameter less than $\varepsilon$. Take $d \in \mathcal{D}$ so that $t_{d} \in V$. Then also $t_{d i} \in V$ for a suitable $i \in\{0,1\}$. Hence $\left\|t_{d}-t_{d i}\right\|_{M}<\varepsilon$. However, from the construction of the sets $U_{d 0}, U_{d 1}$ we have $2 \varepsilon<2 \varepsilon^{\prime \prime}<\left\langle h_{d}, t_{d 0}-t_{d 1}\right\rangle=2\left|\left\langle h_{d}, t_{d}-t_{d i}\right\rangle\right|<$ $2\left\|t_{d}-t_{d i}\right\|_{M}$, a contradiction. Therefore $B_{X^{*}}$ is weak* $2 \varepsilon^{\prime}$ - $M$-fragmentable. Proposition 5 then finishes the proof.

Proposition 7. Let $(X,\|\cdot\|)$ be a Banach space, $M \subset B_{X}$ a non-empty set, and let $\varepsilon>0$ be given. Then (i) $\Rightarrow($ ii) $\Rightarrow$ (iii) where

(i) $B_{X^{*}}$ is weak $k^{*} \varepsilon-M$-fragmentable.

(ii) $M$ is an $\varepsilon$-Asplund set.

(iii) $B_{X^{*}}$ is weak $2 \varepsilon-M$-fragmentable.

Proof. (i) $\Rightarrow$ (ii). We follow the argument from [24, p. 742]. Fix any countable set $A \subset M$. By Zorn's lemma we find a set $S \subset B_{X^{*}}$ such that $\left\|x_{1}^{*}-x_{2}^{*}\right\|_{A} \geq \varepsilon$ for all distinct $x_{1}^{*}, x_{2}^{*} \in S$, and for every $x^{*} \in B_{X^{*}}$ there is $s \in S$ so that $\left\|x^{*}-s\right\|_{A}<\varepsilon$. Assume that $S$ is uncountable. On $B_{X^{*}}$, consider the topology of pointwise convergence on elements of $A-$ call it $\tau_{A}$. Clearly, $\tau_{A}$ is semimetrizable. By deleting at most countably many points from $S$ we get an (uncountable) set $S_{0} \subset S$ such that each point of $S_{0}$ is a $\tau_{A}$-accumulation point of $S_{0}$. This can be done easily by using the concept of condensation points (see $\left[6\right.$, p. 85]). Since $B_{X^{*}}$ is weak ${ }^{*} \varepsilon-M$-fragmentable, we can find a weak* open set $W \subset X^{*}$ such that $S_{0} \cap W$ is non-empty and 
has $M$-diameter less than $\varepsilon$. But this leads to a contradiction because $S_{0} \cap W$ is not a singleton. Therefore $S$ must be at most countable and hence $B_{X^{*}}$

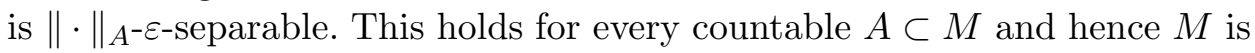
an $\varepsilon$-Asplund set.

(ii) $\Rightarrow$ (iii). We guess that this argument goes back to I. Namioka. Let $U \subset B_{X^{*}}$ be a non-empty set. Let $\{0\} \neq Y_{0}$ be some fixed separable subspace of $X$. Let $W_{i}^{0} \subset B_{X^{*}}, i \in \mathbb{N}$, be a basis for the topology on $B_{X^{*}}$ of pointwise convergence on $Y_{0}$. For every $i \in \mathbb{N}$ we find a countable set $A_{i}^{0} \subset M$ such that $A_{i}^{0}$-diam $\left(U \cap W_{i}^{0}\right)=M$-diam $\left(U \cap W_{i}^{0}\right)$. Let $Y_{1}$ be the closed linear span of $Y_{0} \cup \bigcup_{i \in \mathbb{N}} A_{i}^{0}$. Generally, consider any fixed $n \in \mathbb{N}$ and assume we have already found separable subspaces $Y_{0} \subset Y_{1} \subset \cdots$ $\cdots \subset Y_{n} \subset X$, sets $A_{i}^{0}, A_{i}^{1}, \ldots, A_{i}^{n-1} \subset M, i \in \mathbb{N}$, and relatively weak* open sets $W_{i}^{0}, W_{i}^{1}, \ldots, W_{i}^{n-1} \subset B_{X^{*}}, i \in \mathbb{N}$. Let $W_{i}^{n} \subset B_{X^{*}}, i \in \mathbb{N}$, be a basis for the topology on $B_{X^{*}}$ of pointwise convergence on $Y_{n}$. For every $i \in \mathbb{N}$ we find a countable set $A_{i}^{n} \subset M$ such that $A_{i}^{n}$ - $\operatorname{diam}\left(U \cap W_{i}^{n}\right)=M$ - $\operatorname{diam}\left(U \cap W_{i}^{n}\right)$. Let then $Y_{n+1}$ be the closed linear span of the set $Y_{n} \cup \bigcup_{i \in \mathbb{N}} A_{i}^{n}$. Finally, let $Y$ be the closure of $\bigcup_{i \in \mathbb{N}} Y_{n}$, and put $A=\bigcup_{i, n \in \mathbb{N}} A_{i}^{n}$. We observe that $Y$ is separable and $A$ is countable.

Now, we show that $M-\operatorname{diam}(U \cap W)<2 \varepsilon$ for a suitable weak* open set $W \subset X^{*}$. From (ii) we find a countable set $C \subset B_{X^{*}}$ such that for every $x^{*} \in$ $B_{X^{*}}$ there is $c \in C$ satisfying $\left\|x^{*}-c\right\|_{A}<\varepsilon$. Let $\tau$ denote the (possibly nonHausdorff) topology on $B_{X^{*}}$ of pointwise convergence on $Y$, and let $\bar{U}^{\tau}$ be the closure of $U$ in $\tau$. We note that $\left(B_{X^{*}}, \tau\right)$ is a compact space. We can write

$$
\bar{U}^{\tau}=\bigcup_{c \in C} \bigcup_{j \in \mathbb{N}}\left\{x^{*} \in \bar{U}^{\tau} ;\left\|x^{*}-c\right\|_{A} \leq \varepsilon-1 / j\right\},
$$

and Baire's category theorem yields $c \in C, j \in \mathbb{N}$, and a $\tau$-open set $V \subset B_{X^{*}}$ so that $\emptyset \neq \bar{U}^{\tau} \cap V \subset\left\{x^{*} \in \bar{U}^{\tau} ;\left\|x^{*}-c\right\|_{A} \leq \varepsilon-1 / j\right\}$. Therefore the (non-empty) set $U \cap V$ has $A$-diameter $\leq 2(\varepsilon-1 / j)<2 \varepsilon$. We may and do assume that $V=\left\{x^{*} \in B_{X^{*}} ;\left\langle y_{i}, x^{*}\right\rangle<\alpha_{i}, i=1, \ldots, l\right\}$ for suitable $n, l \in \mathbb{N}, y_{1}, \ldots, y_{l} \in Y_{n}$, and $\alpha_{1}, \ldots, \alpha_{l} \in \mathbb{R}$. Thus $V$ is an open set in the topology of pointwise convergence on $Y_{n}$. Hence, there must exist $i \in \mathbb{N}$ such that $\emptyset \neq U \cap W_{i}^{n} \subset U \cap V$. Find a weak* open set $W \subset X^{*}$ such that $W_{i}^{n}=W \cap B_{X^{*}}$. Then $U \cap W=U \cap W_{i}^{n}$ and hence

$$
\begin{aligned}
M-\operatorname{diam}(U \cap W) & =A_{i}^{n}-\operatorname{diam}\left(U \cap W_{i}^{n}\right) \leq A-\operatorname{diam}\left(U \cap W_{i}^{n}\right) \\
& \leq A-\operatorname{diam}(U \cap V)<2 \varepsilon .
\end{aligned}
$$

REMARK. It is natural to ask what happens if the whole unit ball $B_{X}$ is an $\varepsilon$-Asplund set. Clearly, this information is vacuous if $\varepsilon>1$. On the other hand, if $0<\varepsilon<1$ and $B_{X}$ is an $\varepsilon$-Asplund set, then $X$ is already an Asplund space; this easily follows from Riesz' lemma. Thus we get from Proposition 7 a result of M. Muñoz [22]: If $B_{X^{*}}$ is weak $k^{*} \varepsilon-B_{X}$-fragmentable, i.e., weak* 
$\varepsilon$-fragmentable by the norm, for some $0<\varepsilon<1$, then $X$ is an Asplund space (and hence $B_{X^{*}}$ is weak fragmentable by the norm). Further, using this fact and Proposition 6 we find that if $B_{X^{*}}$ is $\varepsilon$ - $B_{X}$-dentable for some $0<\varepsilon<1 / 2$, then $X$ must be an Asplund space. Finally, if $B_{X}$ is 1-Asplund, then $X$ may not be an Asplund space. In other words, the $\|\cdot\|$-1-separability of $B_{X^{*}}$ may not be enough for the separability of $X^{*}$. The following example illustrating this is due to J. Tišer. We thank him for allowing us to include it in our paper.

Example. Consider $X=\ell_{1}$. Then $X^{*}=\ell_{\infty}$. Endow $X^{*}$ with the (equivalent dual) norm

$$
\left\|\left|x^{*}\right|\right\|\left|=\sup _{n \in \mathbb{N}}\right| x_{n}\left|+\sum_{n=1}^{\infty} 2^{-n}\right| x_{n} \mid, \quad x^{*}=\left(x_{1}, x_{2}, \ldots\right) \in \ell_{\infty} .
$$

Put $e_{1}=(1,0,0, \ldots), e_{2}=(0,1,0, \ldots), \ldots$ We shall show that the closed unit ball in $\left(\ell_{\infty},|\|\cdot \mid\|)\right.$ can be covered by a countable family of open balls of $|\|\cdot\||$-radius 1 . For this we shall show that the open balls $\left\{x^{*} \in \ell_{\infty} ;\left\|x^{*}-\frac{1}{2} e_{m} \mid\right\|\right.$ $<1\}$ and $\left\{x^{*} \in \ell_{\infty} ;\left\|x^{*}+\frac{1}{2} e_{m} \mid\right\|<1\right\}, m \in \mathbb{N}$, cover the unit sphere $\left\{x^{*} \in \ell_{\infty} ;\left\|x^{*} \mid\right\|=1\right\}$. Hence, all these balls, together with the open ball $\left\{x^{*} \in \ell_{\infty} ;\left\|x^{*}-0 \mid\right\|<1\right\}$, will cover the whole closed unit ball $\left\{x^{*} \in \ell_{\infty}\right.$; $\left.\left\|x^{*} \mid\right\| \leq 1\right\}$. So fix any $x^{*}=\left(x_{1}, x_{2}, \ldots\right) \in \ell_{\infty}$ with $\left\|x^{*}\right\| \mid=1$. Define $\left\|x^{*}\right\|_{\infty}=\sup _{n \in \mathbb{N}}\left|x_{n}\right|$. Then $1 \leq\left\|x^{*}\right\|_{\infty}+\sum_{n=1}^{\infty} 2^{-n}\left\|x^{*}\right\|_{\infty}=2\left\|x^{*}\right\|_{\infty}$, and so $\left\|x^{*}\right\|_{\infty} \geq 1 / 2$. Assume first that $\left\|x^{*}\right\|_{\infty}>1 / 2$. Find $m \in \mathbb{N}$ so that $\left|x_{m}\right|>1 / 2$. Assume, say, $x_{m}>0$; then $x_{m}>1 / 2$. We shall show that ||$\left|x^{*}-\frac{1}{2} e_{m}\right|||<1$. Indeed,

$$
\begin{aligned}
|| \mid x^{*}- & \frac{1}{2} e_{m}|\|=| x_{m}-1 / 2\left|\vee \sup _{n \neq m}\right| x_{n}\left|+2^{-m}\right| x_{m}-1 / 2\left|+\sum_{n \neq m} 2^{-n}\right| x_{n} \mid \\
& =\left(x_{m}-1 / 2\right) \vee \sup _{n \neq m}\left|x_{n}\right|+2^{-m}\left(x_{m}-1 / 2\right)+\left(1-2^{-m}\left|x_{m}\right|-\left\|x^{*}\right\|_{\infty}\right) \\
& \leq\left\|x^{*}\right\|_{\infty}+2^{-m} x_{m}-2^{-m-1}+1-2^{-m} x_{m}-\left\|x^{*}\right\|_{\infty}<1 .
\end{aligned}
$$

If $x_{m}<0$, then we find similarly that $\mid\left\|x^{*}+\frac{1}{2} e_{m}\right\|<1$. Second, assume that $\left\|x^{*}\right\|_{\infty}=1 / 2$. Then, necessarily, $\left|x_{n}\right|=1 / 2$ for every $n \in \mathbb{N}$ and so

$$
\min \left\|\mid x^{*} \pm \frac{1}{2} e_{1}\right\|=\frac{1}{2}+\sum_{n=2}^{\infty} 2^{-n} \frac{1}{2}=\frac{3}{4}<1 .
$$

QUESTION. If $X$ is a general separable Banach space with non-separable dual, does it admit an equivalent norm $|\| \cdot|||$ such that the closed unit ball $B_{(X,\|\cdot\|)}$ is a 1 -Asplund set?

Next, we shall focus on $\varepsilon$-variants of some concepts of smoothness and rotundity of the norm. An example of a space with a Gateaux smooth norm 
that has no equivalent $M$-smooth norm, with $M$ linearly dense, is any nonWCG subspace of a WCG space (see, e.g., [9, Theorem 1]).

Proposition 8. Let $(X,\|\cdot\|)$ be a Banach space, $M \subset B_{X}$ a non-empty set, and let $\varepsilon>0$ be given. Then (i) $\Rightarrow($ ii) $\Rightarrow($ iii) where

(i) The dual norm $\|\cdot\|$ on $X^{*}$ is $\varepsilon-M-L U R$.

(ii) The dual norm $\|\cdot\|$ on $X^{*}$ has the weak $k^{*} \varepsilon-M$-Kadec property.

(iii) The set $M$ is $\varepsilon$-Asplund.

Proof. (i) $\Rightarrow$ (ii). Let $x^{*}$ and a net $\left(x_{\tau}^{*}\right)_{\tau \in T}$ lie in $S_{X^{*}}$ and $x_{\tau}^{*} \stackrel{w^{*}}{\rightarrow} x^{*}$. Then for every $x \in B_{X}$ we have

$$
2 \geq \limsup _{\tau}\left\|x^{*}+x_{\tau}^{*}\right\| \geq \liminf _{\tau}\left\|x^{*}+x_{\tau}^{*}\right\| \geq \lim _{\tau}\left\langle x, x^{*}+x_{\tau}^{*}\right\rangle=2\left\langle x, x^{*}\right\rangle .
$$

Hence $\lim _{\tau}\left\|x^{*}+x_{\tau}^{*}\right\|=2$. Assume that $\lim \sup _{\tau}\left\|x_{\tau}^{*}-x^{*}\right\|_{M} \geq \varepsilon$. Find then $\tau_{1}<\tau_{2}<\cdots$ in $T$ such that

$$
\left\|x_{\tau_{n}}^{*}-x^{*}\right\|_{M}>\varepsilon-1 / n \text { and }\left\|x_{\tau_{n}}^{*}+x^{*}\right\|>2-1 / n
$$

for every $n \in \mathbb{N}$. Then $\lim _{n \rightarrow \infty}\left\|x_{\tau_{n}}^{*}+x^{*}\right\|=2$, and therefore, by (i), $\lim \sup _{n \rightarrow \infty}\left\|x_{\tau_{n}}^{*}-x^{*}\right\|_{M}<\varepsilon$, a contradiction.

(ii) $\Rightarrow$ (iii). Let $\emptyset \neq A \subset M$ be a countable subset. Denote by $Y$ the closed linear span of $A$ and let $Q: X^{*} \rightarrow Y^{*}$ be the canonical quotient mapping. Let $\tau$ denote the topology, on the dual unit sphere $S_{X^{*}}$, of pointwise convergence on elements of $A$. Then $\left(S_{X^{*}}, \tau\right)$ is a separable space (maybe not Hausdorff). Find a countable set $D \subset S_{X^{*}}$ which is $\tau$-dense in $S_{X^{*}}$. Fix any $0 \neq y^{*}$ $\in B_{Y^{*}}$. Find a sequence $\left(d_{n}\right)$ in $D$ such that $\left\langle x, Q\left(d_{n}\right)\right\rangle \rightarrow\left\langle x, y^{*} /\left\|y^{*}\right\|\right\rangle$ as $n \rightarrow \infty$ for every $x \in A$. Let $x^{*} \in X^{*}$ be a weak* cluster point of $\left(d_{n}\right)$. It is easy to see that $\left\|x^{*}\right\|=1$. From (ii) we can then find $n \in \mathbb{N}$ so that $\left\|x^{*}-d_{n}\right\|_{M}<\varepsilon$, and hence

$$
\left\|y^{*} /\right\| y^{*}\left\|-Q\left(d_{n}\right)\right\|_{A}=\left\|x^{*}-d_{n}\right\|_{A} \leq\left\|x^{*}-d_{n}\right\|_{M}<\varepsilon .
$$

Therefore $\left\|y^{*}-\right\| y^{*}\left\|Q\left(d_{n}\right)\right\|_{A}<\left\|y^{*}\right\| \varepsilon \leq \varepsilon$. Thus, the (countable) set $\bigcup\{r Q(D) ; r \in \mathbb{Q}, 0 \leq r \leq 1\}$, where $\mathbb{Q}$ stands for the set of rational numbers, witnesses that $B_{Y^{*}}$ is $\|\cdot\|_{A^{-}-\varepsilon \text {-separable. Finally, the (countable) }}$ set $\bigcup\{r D ; r \in \mathbb{Q}, 0 \leq r \leq 1\}$, witnesses that $B_{X^{*}}$ is $\|\cdot\|_{A^{-}-\varepsilon \text {-separable. We }}$ have thus proved (iii).

We also have the following variant of Proposition 8.

Proposition 9. Let $(X,\|\cdot\|)$ be a Banach space, $M \subset B_{X}$ a non-empty set, and let $\varepsilon>0$ be given. Then (i) $\Rightarrow($ ii $) \Rightarrow$ (iii) where

(i) The dual norm $\|\cdot\|$ on $X^{*}$ is $\frac{1}{2} \varepsilon-M-L U R$.

(ii) The norm $\|\cdot\|$ on $X$ is $\varepsilon$-M-smooth.

(iii) The set $M$ is $\varepsilon^{\prime}$-Asplund for every $\varepsilon^{\prime}>\varepsilon$. 
Proof. (i) $\Rightarrow$ (ii). Consider any $x_{0} \in S_{X}$. Let $\left(x_{n}^{*}\right)$ and $\left(y_{n}^{*}\right)$ be two sequences in $B_{X^{*}}$ such that $\left\langle x_{0}, x_{n}^{*}\right\rangle \rightarrow 1$ and $\left\langle x_{0}, y_{n}^{*}\right\rangle \rightarrow 1$. Find $x_{0}^{*} \in S_{X^{*}}$ such that $\left\langle x_{0}, x_{0}^{*}\right\rangle=1$. Then

$$
2 \geq \limsup _{n \rightarrow \infty}\left\|x_{0}^{*}+x_{n}^{*}\right\| \geq \liminf _{n \rightarrow \infty}\left\|x_{0}^{*}+x_{n}^{*}\right\| \geq \lim _{n \rightarrow \infty}\left\langle x_{0}, x_{0}^{*}+x_{n}^{*}\right\rangle=2,
$$

and similarly, $\lim _{n \rightarrow \infty}\left\|x_{0}^{*}+y_{n}^{*}\right\|=2$. Thus (i) implies that

$$
\limsup _{n \rightarrow \infty}\left\|x_{0}^{*}-x_{n}^{*}\right\|_{M}<\varepsilon / 2 \text { and } \quad \limsup _{n \rightarrow \infty}\left\|x_{0}^{*}-y_{n}^{*}\right\|_{M}<\varepsilon / 2 \text {. }
$$

Then $\lim \sup _{n \rightarrow \infty}\left\|x_{n}^{*}-y_{n}^{*}\right\|_{M}<\varepsilon$. Now, Proposition 4 yields (ii).

(ii) $\Rightarrow$ (iii). Take any countable subset $\emptyset \neq A \subset M$. Denote by $Y$ the closed linear span of $A$; this subspace is separable. Find a countable dense subset $C$ in $S_{Y}$. For every $c \in C$ we find $c^{*} \in S_{Y^{*}}$ such that $\left\langle c, c^{*}\right\rangle=1$, and let $C^{*}$ denote the set of all such $c^{*}$ 's. Now, fix any $y^{*} \in S_{Y^{*}}$ such that there is $y \in S_{Y}$ satisfying $\left\langle y, y^{*}\right\rangle=1$. Find a sequence $\left(c_{n}\right)$ in $C$ norm converging to $y$. For every $n \in \mathbb{N}$ find $c_{n}^{*} \in C^{*}$ such that $\left\langle c_{n}, c_{n}^{*}\right\rangle=1$. We have

$$
\left\langle y, c_{n}^{*}\right\rangle=\left\langle c_{n}, c_{n}^{*}\right\rangle+\left\langle y-c_{n}, c_{n}^{*}\right\rangle=1+\left\langle y-c_{n}, c_{n}^{*}\right\rangle \rightarrow 1 \quad \text { as } n \rightarrow \infty .
$$

Hence, by Proposition 4 , there is $n \in \mathbb{N}$ so that $\left\|c_{n}^{*}-y^{*}\right\|_{A} \leq\left\|c_{n}^{*}-y^{*}\right\|_{M \cap Y}$ $<\varepsilon$. We have proved that the set of all norm attaining elements of $S_{Y^{*}}$ is

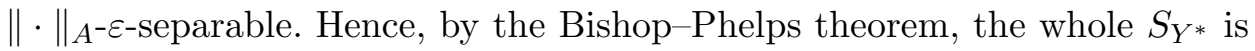
$\|\cdot\|_{A^{-}} \varepsilon^{\prime}$-separable for every $\varepsilon^{\prime}>\varepsilon$. Then the (countable) set $D:=\bigcup\left\{r C^{*}\right.$; $r \in \mathbb{Q}, 0 \leq r \leq 1\}$ shows that the whole ball $B_{Y^{*}}$ is $\|\cdot\|_{A^{-}-\varepsilon^{\prime} \text {-separable for }}$ every $\varepsilon^{\prime}>\varepsilon$. Finally, extending every element of $d \in D$ to $\tilde{d} \in B_{X^{*}}$, the (countable) set $\{\tilde{d} ; d \in D\}$ witnesses that $B_{X^{*}}$ is $\|\cdot\|_{A^{-} \varepsilon^{\prime} \text {-separable for every }}$ $\varepsilon^{\prime}>\varepsilon$. We have thus proved (iii).

If $X^{*}$ is separable, then it admits an equivalent dual LUR norm [4, Corollary II.4.3]. A quantitative version of this, which can be thought of as a kind of converse to Propositions 8 and 9, reads:

Proposition 10. Let $(X,\|\cdot\|)$ be a Banach space, let $\Delta>0, \varepsilon>0$ be given, and let $\emptyset \neq M \subset B_{X}$ be a separable $\varepsilon$-Asplund set. Then $X$ admits an equivalent norm $|\cdot|$, with $|\cdot| \leq\|\cdot\| \leq(1+\Delta)|\cdot|$, whose dual norm is $2 \varepsilon-M-L U R$.

Proof. We use Godefroy's method of transfer ([4, Ch. II $])$. Replace $M$ by $M \cup(-M)$ and call it again $M$; this set will also be $\varepsilon$-Asplund. Find $d_{i}^{*} \in B_{X^{*}}, i \in \mathbb{N}$, such that for every $x^{*} \in B_{X^{*}}$ there is $j \in \mathbb{N}$ so that $\left\|x^{*}-d_{j}^{*}\right\|_{M}<\varepsilon$. For $k \in \mathbb{N}$ define

$$
\left|x^{*}\right|_{k}^{2}:=\inf \left\{\left\|x^{*}-\sum_{i=1}^{\infty} \frac{1}{i} y_{i} d_{i}^{*}\right\|_{M}^{2}+\frac{1}{k} \sum_{i=1}^{\infty} y_{i}{ }^{2} ;\left(y_{i}\right) \in \ell_{2}\right\}, \quad x^{*} \in X^{*}
$$


and then

$$
\left|x^{*}\right|^{2}:=\left\|x^{*}\right\|^{2}+\Delta \sum_{k=1}^{\infty} 2^{-k}\left|x^{*}\right|_{k}^{2}, \quad x^{*} \in X^{*} .
$$

Clearly $\left\|x^{*}\right\|^{2} \leq\left|x^{*}\right|^{2} \leq(1+\Delta)\left\|x^{*}\right\|^{2}$ for every $x^{*} \in X^{*}$. A use of the triangle inequality in $\ell_{2}$ reveals that $|\cdot|$ is subadditive. That $|\cdot|$ is weak* lower semicontinuous can be proved similarly to [4, Lemma VII.2.5(i)]. Therefore $|\cdot|$ is an equivalent dual norm on $X^{*}$. We denote by $|\cdot|$ also the norm on $X$ predual to $|\cdot|$; then $|x|^{2} \leq\|x\|^{2} \leq(1+\Delta)|x|^{2}$ for every $x \in X$.

Now, consider $x_{0}^{*}, x_{1}^{*}, x_{2}^{*}, \ldots$ in the unit sphere of $\left(X^{*},|\cdot|\right)$ such that $\left|x_{0}^{*}+x_{n}^{*}\right| \rightarrow 2$ as $n \rightarrow \infty$. We have to show that $\lim \sup _{n \rightarrow \infty}\left\|x_{0}^{*}-x_{n}^{*}\right\|_{M}$ $<2 \varepsilon$. Find $j \in \mathbb{N}$ so that $\left\|x_{0}^{*}-d_{j}^{*}\right\|_{M}<\varepsilon$. Then find $k \in \mathbb{N}$ so large that $\left\|x_{0}^{*}-d_{j}^{*}\right\|_{M}^{2}+j^{2} / k<\varepsilon^{2}$. Further, for $n=0,1,2, \ldots$ find $y_{n}=\left(y_{1}^{n}, y_{2}^{n}, \ldots\right) \in \ell_{2}$ such that

$$
\left|x_{n}^{*}\right|_{k}^{2}=\left\|x_{n}^{*}-\sum_{i=1}^{\infty} \frac{1}{i} y_{i}^{n} d_{i}^{*}\right\|_{M}^{2}+\frac{1}{k} \sum_{i=1}^{\infty}\left(y_{i}^{n}\right)^{2} ;
$$

this is possible because the mapping $\Phi$ from $\ell_{2}$ with weak topology into $\mathbb{R}$ defined for $y=\left(y_{i}\right) \in \ell_{2}$ by

$$
\Phi(y):=\left\|x^{*}-\sum_{i=1}^{\infty} \frac{1}{i} y_{i} d_{i}^{*}\right\|_{M}^{2}+\frac{1}{k} \sum_{i=1}^{\infty} y_{i}^{2}
$$

is lower semicontinuous and $\Phi\left(z_{m}\right) \rightarrow \infty$ whenever $z_{1}, z_{2}, \ldots \in \ell_{2}$ and $\left\|z_{m}\right\|_{2} \rightarrow \infty$. We have the estimate

$$
\begin{aligned}
\left\|x_{0}^{*}-x_{n}^{*}\right\|_{M} \leq & \left\|x_{0}^{*}-\sum_{i=1}^{\infty} \frac{1}{i} y_{i}^{0} d_{i}^{*}\right\|_{M}+\left\|\sum_{i=1}^{\infty} \frac{1}{i}\left(y_{i}^{0}-y_{i}^{n}\right) d_{i}^{*}\right\|_{M} \\
& +\left\|\sum_{i=1}^{\infty} \frac{1}{i} y_{i}^{n} d_{i}^{*}-x_{n}^{*}\right\|_{M} \\
\leq & \left|x_{0}^{*}\right|_{k}+\left(\sum_{i=1}^{\infty} \frac{1}{i^{2}}\right)^{1 / 2}\left(\sum_{i=1}^{\infty}\left(y_{i}^{0}-y_{i}^{n}\right)^{2}\right)^{1 / 2}+\left|x_{n}^{*}\right|_{k}
\end{aligned}
$$

for every $n=1,2, \ldots$ Now, a convexity argument and the parallelogram identity in $\ell_{2}$ yield, for every $n \in \mathbb{N}$,

$$
4-\left|x_{0}^{*}+x_{n}^{*}\right|^{2} \geq \Delta 2^{-k}\left(\left|x_{0}^{*}\right|_{k}-\left|x_{n}^{*}\right|_{k}\right)^{2}
$$

and

$$
4-\left|x_{0}^{*}+x_{n}^{*}\right|^{2} \geq \Delta 2^{-k} \frac{1}{k} \sum_{i=1}^{\infty}\left(y_{i}^{0}-y_{i}^{n}\right)^{2} .
$$


Hence, because $\lim _{n \rightarrow \infty}\left|x_{0}^{*}+x_{n}^{*}\right|=2$, we have

$$
\left|x_{n}^{*}\right|_{k} \rightarrow\left|x_{0}^{*}\right|_{k} \quad \text { and } \quad \sum_{i=1}^{\infty}\left(y_{i}^{0}-y_{i}^{n}\right)^{2} \rightarrow 0 \quad \text { as } n \rightarrow \infty \text {. }
$$

Therefore, (1) shows that $\limsup _{n \rightarrow \infty}\left\|x_{0}^{*}-x_{n}^{*}\right\|_{M} \leq 2\left|x_{0}^{*}\right|_{k}$. As

$$
\left|x_{0}^{*}\right|_{k}^{2} \leq\left\|x_{0}^{*}-\frac{1}{j} j d_{j}^{*}\right\|_{M}^{2}+\frac{1}{k} j^{2}=\left\|x_{0}^{*}-d_{j}^{*}\right\|_{M}^{2}+\frac{j^{2}}{k}<\varepsilon^{2},
$$

we conclude that $\limsup _{n \rightarrow \infty}\left\|x_{0}^{*}-x_{n}^{*}\right\|_{M}<2 \varepsilon$.

Next, we focus on $\varepsilon$-weak compactness. Given $\varepsilon>0$, we say that a subset $M$ of a Banach space $(X,\|\cdot\|)$ is $\varepsilon$-weakly compact if it is bounded and $\bar{M}^{*} \subset X+\varepsilon^{\prime} B_{X^{* *}}$ for every $\varepsilon^{\prime}>\varepsilon$, or equivalently, $\operatorname{dist}\left(x^{* *}, X\right) \leq \varepsilon$ for every $x^{* *} \in \bar{M}^{*}$. A simple example of such a set is of course $K+\varepsilon B_{X}$, where $K$ is a weakly compact subset of $X$. However, not all $\varepsilon$-weakly compact sets are of this nature (see [15, Remark 4]). For another example, coming naturally from uniform Gateaux smoothness, see [10]. Some results related to this concept are presented in [12] and [17]. Clearly, if a set is $\varepsilon$-weakly compact for every $\varepsilon>0$, then it is relatively weakly compact.

Proposition 11. Let $(X,\|\cdot\|)$ be a Banach space, $M \subset B_{X}$ a non-empty set, and let $\varepsilon>0$ be given. Then (i) $\Rightarrow(\mathrm{ii}) \Rightarrow($ iii) where

(i) The double dual norm $\|\cdot\|$ on $X^{* *}$ is $\varepsilon$-M-smooth.

(ii) $M$ is $\varepsilon$-weakly compact.

(iii) $M$ is $4 \varepsilon^{\prime}$-Asplund for every $\varepsilon^{\prime}>\varepsilon$.

Proof. (i) $\Rightarrow\left(\right.$ ii). The argument is from [10]. Take an arbitrary $x^{* *} \in \bar{M}^{*}$. Put $d=\operatorname{dist}\left(x^{* *}, X\right)$. Assume that $d>0$. By the Hahn-Banach theorem find $F \in X^{* * *}$ with $\|F\|=1$ such that $F$ vanishes on $X$ and $\left\langle x^{* *}, F\right\rangle=d$. Fix any $\varepsilon^{\prime}>\varepsilon$. From the Bishop-Phelps theorem find $G \in X^{* * *}$ and $x_{0}^{* *} \in X^{* *}$ such that $\|G-F\|<\frac{1}{2}\left(\varepsilon^{\prime}-\varepsilon\right)$ and $\left\langle x_{0}^{* *}, G\right\rangle=1=\left\|x_{0}^{* *}\right\|=\|G\|$. Using Goldstine's theorem we find a sequence $\left(x_{k}^{*}\right)$ in $B_{X *}$ so that

$$
\left\langle x_{0}^{* *}, x_{k}^{*}\right\rangle \rightarrow\left\langle x_{0}^{* *}, G\right\rangle \quad \text { and } \quad\left\langle x^{* *}, x_{k}^{*}\right\rangle \rightarrow\left\langle x^{* *}, G\right\rangle \quad \text { as } k \rightarrow \infty .
$$

Since the double dual norm $\|\cdot\|$ on $X^{* *}$ is $\varepsilon$ - $M$-differentiable at $x_{0}^{* *}$, Proposition 4 shows that $\lim \sup _{k \rightarrow \infty}\left\|x_{k}^{*}-G\right\|_{M}<\varepsilon$. But $F$ vanishes on $X$; so

$$
\begin{aligned}
& \limsup _{k \rightarrow \infty}\left\|x_{k}^{*}\right\|_{M}=\limsup _{k \rightarrow \infty}\left\|x_{k}^{*}-F\right\|_{M} \\
& \quad \leq \limsup _{k \rightarrow \infty}\left\|x_{k}^{*}-G\right\|_{M}+\|G-F\|_{M}<\varepsilon+\frac{1}{2}\left(\varepsilon^{\prime}-\varepsilon\right)=\frac{1}{2}\left(\varepsilon^{\prime}+\varepsilon\right) .
\end{aligned}
$$

Hence $\lim \sup _{k \rightarrow \infty}\left\langle x^{* *}, x_{k}^{*}\right\rangle<\frac{1}{2}\left(\varepsilon^{\prime}+\varepsilon\right)$, and so $\left\langle x^{* *}, G\right\rangle<\frac{1}{2}\left(\varepsilon^{\prime}+\varepsilon\right)$. Now $\operatorname{dist}\left(x^{* *}, X\right)=\left\langle x^{* *}, F\right\rangle=\left\langle x^{* *}, G\right\rangle+\left\langle x^{* *}, F-G\right\rangle\left\langle\frac{1}{2}\left(\varepsilon^{\prime}+\varepsilon\right)+\frac{1}{2}\left(\varepsilon^{\prime}-\varepsilon\right)=\varepsilon^{\prime}\right.$. Since $\varepsilon^{\prime}>\varepsilon$ was arbitrary, we get (ii). 
(ii) $\Rightarrow$ (iii). Let $\emptyset \neq A \subset M$ be a countable set. Find a countable set $D \subset B_{X^{*}}$ which is dense in $B_{X^{*}}$ in the topology of pointwise convergence on elements of $A$. We shall show that $B_{X^{*}}$ lies in the norm closure of the set co $D+4 \varepsilon A^{\circ}$, where co $D$ is the convex hull of $D$ and $A^{\circ}=\left\{x^{*} \in X^{*}\right.$; $\left.\sup \left|\left\langle A, x^{*}\right\rangle\right| \leq 1\right\}$. Assume that this is false. Find then $x^{*} \in B_{X^{*}}$ and $F \in X^{* *}$ so that

$$
\left\langle F, x^{*}\right\rangle>\sup \left\langle F, \operatorname{co} D+4 \varepsilon A^{\circ}\right\rangle \quad\left(=\sup \langle F, D\rangle+4 \varepsilon \sup \left\langle F, A^{\circ}\right\rangle\right) .
$$

As $A \subset B_{X}$, we may and do assume that $\sup \left\langle F, A^{\circ}\right\rangle=1$; thus $\left\langle F, x^{*}\right\rangle>$ $\sup \langle F, D\rangle+4 \varepsilon$. Now, the bipolar theorem implies that $F \in \overline{\operatorname{co}(A \cup-A)} *$ $\left(\subset \overline{\operatorname{co}(M \cup-M)}^{*} \subset X^{* *}\right)$. But, by [12], $\overline{\operatorname{co}(M \cup-M)}^{*}$ is $2 \varepsilon$-weakly compact. Hence $\overline{\operatorname{co}(A \cup-A)}^{*} \subset X+2 \varepsilon^{\prime} B_{X^{* *}}$ for every $\varepsilon^{\prime}>\varepsilon$. Take

$$
\varepsilon<\delta<\frac{1}{4}\left(\left\langle F, x^{*}\right\rangle-\sup \langle F, D\rangle\right) .
$$

We can write $F=x+x^{* *}$ where $x \in X, x^{* *} \in X^{* *}$, and $\left\|x^{* *}\right\|<2 \delta$. Thus

$$
\begin{aligned}
\left\langle x, x^{*}\right\rangle+2 \delta & >\left\langle x+x^{* *}, x^{*}\right\rangle=\left\langle F, x^{*}\right\rangle>\sup \langle F, D\rangle+4 \delta \\
& \geq \sup \langle x, D\rangle-2 \delta+4 \delta=\|x\|+2 \delta \geq\left\langle x, x^{*}\right\rangle+2 \delta,
\end{aligned}
$$

a contradiction. Thus we have shown that $B_{X^{*}}$ is a subset of the norm closure of $\operatorname{co} D+4 \varepsilon A^{\circ}$. Now, let $C$ be a countable dense subset of co $D$. Then $B_{X^{*}} \subset C+4 \varepsilon^{\prime} A^{\circ}$ for every $\varepsilon^{\prime}>\varepsilon$. This proves that $A$ is $4 \varepsilon^{\prime}$-Asplund for every $\varepsilon^{\prime}>\varepsilon$.

Remark. The implication (i) $\Rightarrow$ (ii) in Proposition 11 applied for $M:=$ $B_{X}$ and every $\varepsilon>0$ yields the well-known fact that $(X,\|\cdot\|)$ is reflexive if its norm is uniformly Fréchet smooth. Indeed, the corresponding double dual norm on $X^{* *}$ is then also uniformly Fréchet smooth. Hence $B_{X}$ is $\varepsilon$-weakly compact for every $\varepsilon>0$.

Let us mention two more criteria for $\varepsilon$-weak compactness, which may be of use (see [10]). Given a Banach space $(X,\|\cdot\|)$, and a linear set $Y \subset X^{*}$, the seminorm $x \mapsto \sup \left\langle x, Y \cap B_{X^{*}}\right\rangle, x \in X$, is called the $Y$-envelope of $\|\cdot\|$; clearly, it is lower semicontinuous with respect to the topology of pointwise convergence on $Y$.

Proposition 12. Let $(X,\|\cdot\|)$ be a Banach space, $M \subset B_{X}$ be a nonempty set, and let $\varepsilon>0$ be given. Assume that for every norming hyperplane $Y \subset X^{*}$ the $Y$-envelope of $\|\cdot\|$ is $\varepsilon$-M-smooth. Then $M$ is $2 \varepsilon$-weakly compact.

Proof. Take $x^{* *} \in \bar{M}^{*}$ and assume that $\operatorname{dist}\left(x^{* *}, X\right)>2 \varepsilon$. It is well known that the kernel of $x^{* *}$ - call it $Y$-is a norming hyperplane (see, e.g., [10]). Then the $Y$-envelope of $\|\cdot\|$ - call it $|\cdot|$-is an equivalent norm on $X$. Let $|\cdot|$ also denote the corresponding dual norm on $X^{*}$ and the bidual norm on $X^{* *}$. Observe that the closed unit ball in $\left(X^{*},|\cdot|\right)$ is just $\overline{Y \cap B_{X^{*}}} *$. By [15, Proposition 8], $\left|x^{* *}\right|>\varepsilon$. The Bishop-Phelps theorem yields $x \in X$ 
and $x^{*} \in \overline{Y \cap B_{X^{*}}}{ }^{*}$ so that $\left\langle x, x^{*}\right\rangle=1=|x|=\left|x^{*}\right|$ and $\left\langle x^{* *}, x^{*}\right\rangle>\varepsilon$. Find a sequence $\left(y_{n}^{*}\right)$ in $Y \cap B_{X^{*}}$ such that $\left\langle x, y_{n}^{*}\right\rangle \rightarrow\left\langle x, x^{*}\right\rangle$ as $n \rightarrow \infty$. Since $|\cdot|$ is $\varepsilon$-M-smooth, Proposition 4 yields $n \in \mathbb{N}$ so large that $\left\|x^{*}-y_{n}^{*}\right\|_{M}<\varepsilon$. It then follows that $\left\langle x^{* *}, x^{*}\right\rangle=\left\langle x^{* *}, x^{*}-y_{n}^{*}\right\rangle<\varepsilon$, a contradiction.

For the case of a dual Banach space we can dispense with the condition on norming hyperplanes. Actually, we have the following quantitative version of the well-known fact that $X$ is reflexive if the dual norm on $X^{*}$ is Fréchet smooth.

Proposition 13. Let $(X,\|\cdot\|)$ be a Banach space, let $\varepsilon>0$, let $M$ be a non-empty subset of $B_{X^{*}}$, and assume that the dual norm $\|\cdot\|$ on $X^{*}$ is $\varepsilon-M$-smooth. Then $M$ is $2 \varepsilon$-weakly compact.

Proof. Let $x^{* * *}=x^{*}+x^{\perp} \in \bar{M}^{*}$, where $x^{*} \in X^{*}$ and $x^{\perp} \in X^{\perp}$, and assume $\operatorname{dist}\left(x^{\perp}, X^{*}\right)>2 \varepsilon$. By [15, Proposition 8] applied to $x^{\perp}$, we get $\sup \left\langle\bar{B}_{Y}^{*}, x^{\perp}\right\rangle>\varepsilon$, where $Y \subset X^{* *}$ is the kernel of $x^{\perp}$. Note that $Y$ is then a 1-norming hyperplane in $X^{* *}$ (as it contains $X$ ). It follows that $\bar{B}_{Y}^{*}=B_{X^{* *}}$ and we get $\left\|x^{\perp}\right\|=\sup \left\langle x^{\perp}, \bar{B}_{Y}^{*}\right\rangle>\varepsilon$. The Bishop-Phelps theorem yields $x_{0}^{* *} \in S_{X^{* *}}$ and $x_{0}^{*} \in S_{X^{*}}$ such that $\left\langle x_{0}^{* *}, x_{0}^{*}\right\rangle=1$ and $\left\langle x_{0}^{* *}, x^{\perp}\right\rangle>\varepsilon$. Let $\left(x_{n}\right)$ be a sequence in $B_{X}$ such that $\left\langle x_{0}^{* *}-x_{n}, x_{0}^{*}\right\rangle \rightarrow 0$ and $\left\langle x_{0}^{* *}-x_{n}, x^{*}\right\rangle \rightarrow 0$ as $n \rightarrow \infty$. Then for all large $n \in \mathbb{N}$ we have, by Proposition $4,\left\|x_{0}^{* *}-x_{n}\right\|_{M}<\varepsilon$, and hence $\left\langle x_{0}^{* *}-x_{n}, x^{* * *}\right\rangle<\varepsilon$. Fix any $\delta>0$. Then $\left\langle x_{n}-x_{0}^{* *}, x^{*}\right\rangle<\delta$ for all large $n \in \mathbb{N}$, and hence $\left(\left\langle x_{0}^{* *}, x^{\perp}\right\rangle=\right)\left\langle x_{0}^{* *}-x_{n}, x^{\perp}\right\rangle<\varepsilon+\delta$ for all large $n \in \mathbb{N}$. As $\delta>0$ was arbitrary, we get $\left\langle x_{0}^{* *}, x^{\perp}\right\rangle \leq \varepsilon$, a contradiction. This proves that $\operatorname{dist}\left(x^{\perp}, X^{*}\right) \leq 2 \varepsilon$, that is, $\operatorname{dist}\left(x^{* * *}, X^{*}\right) \leq 2 \varepsilon$.

\section{Proofs of Theorems 1 and 3}

Proof of Theorem 1. The implication $(\mathrm{o}) \Rightarrow(\mathrm{i})$ is proved in [9, p. 438]. The chain $(\mathrm{i}) \Rightarrow($ ii $) \Rightarrow($ iv) follows from Proposition 8 , while (i) $\Rightarrow$ (iii) $\Rightarrow$ (iv) follows from Proposition 9.

It remains to prove (iv) $\Rightarrow(\mathrm{o})$ provided that $X$ is WLD. In the course of the proof, we shall use an $\varepsilon$-variant of the Jayne-Rogers selection theorem due to Stegall, a technique of projectional resolutions of the identity, Simons' lemma, transfinite induction, and a separable reduction.

We start with a selection statement, which is a slight variant of $[7$, Lemma 8.1.1]. Hence, we omit its proof. We recall that $\partial\|\cdot\|$ is the MoreauRockafellar subdifferential of the norm $\|\cdot\|$ (see $[25$, p. 6]).

Proposition 14. Let $(Z,\|\cdot\|)$ be a Banach space, let $\varepsilon>0$, and let $\emptyset \neq M \subset Z$ be a bounded set. Assume that for every non-empty closed set $C \subset Z$ there exist an open set $U \subset Z$ and $\zeta \in B_{Z^{*}}$ such that $C \cap U \neq \emptyset$ and $\|\cdot\|_{M}-\operatorname{dist}(\partial\|\cdot\|(z), \zeta)<\varepsilon$ for every $z \in C \cap U$. Then there exists a Baire 
class one mapping $f: Z \rightarrow\left(Z^{*},\|\cdot\|_{M}\right)$, with $f(Z) \subset B_{Z^{*}}$, such that

$$
\|\cdot\|_{M}-\operatorname{dist}(\partial\|\cdot\|(z), f(z))<\varepsilon \quad \text { for every } z \in Z .
$$

A projectional resolution of the identity (for short $P R I$ ) on a non-separable Banach space $Z$ is a family $\left(P_{\alpha} ; \omega \leq \alpha \leq \mu\right)$ of norm one projections on $Z$, where $\omega$ is the first infinite ordinal and $\mu$ is the first ordinal with cardinality dens $Z$ such that $P_{\omega} \equiv 0, P_{\mu}$ is the identity mapping on $Z$, $P_{\alpha} \circ P_{\beta}=P_{\min \{\alpha, \beta\}}$ for all $\alpha$ and $\beta$ in $[\omega, \mu]$, dens $P_{\alpha}(Z) \leq \# \alpha$ for every $\alpha \in[\omega, \mu]$, and for every $z \in Z$ the mapping $\alpha \mapsto P_{\alpha}(z)$ is continuous from $[\omega, \mu]$ into $Z$. For more information about the PRI, see, e.g., [7, Section 6.1].

Proposition 15. Let $(Z,\|\cdot\|)$ be a non-separable Banach space admitting a linearly dense set $\Gamma \subset B_{Z}$ such that $\#\left\{\gamma \in \Gamma ;\left\langle\gamma, z^{*}\right\rangle \neq 0\right\}$ is at most countable for every $z^{*} \in Z^{*}$ (hence $Z$ is WLD). Assume that for every $n \in \mathbb{N}$ we have $\varepsilon_{n}>0$ and a closed convex symmetric $\varepsilon_{n}$-Asplund set $M_{n} \subset B_{Z}$. Then there exists a PRI $\left(P_{\alpha} ; \omega \leq \alpha \leq \mu\right)$ on $Z$ such that $P_{\alpha}\left(M_{n}\right) \subset M_{n}, P_{\alpha}(\gamma) \in\{\gamma, 0\}$ for every $\alpha \in[\omega, \mu]$, every $n \in \mathbb{N}$, and every $\gamma \in \Gamma$, and moreover, for every limit ordinal $\omega<\lambda \leq \mu$, every $n \in \mathbb{N}$, and every $z^{*} \in B_{Z^{*}}$ we have

$$
\limsup _{\beta \uparrow \lambda}\left\|P_{\lambda}^{*} z^{*}-P_{\beta}^{*} z^{*}\right\|_{M_{n}}<9 \varepsilon_{n}
$$

Proof. We elaborate the argument from [16], which goes back to [19]. Fix any $n \in \mathbb{N}$. We verify the assumptions of Proposition 14 . So take any closed set $\emptyset \neq C \subset Z$. Let $F: C \rightarrow 2^{\left(B_{Z^{*}}, w^{*}\right)}$ be a minimal usco mapping such that $F(z) \subset \partial\|\cdot\|(z)$ for every $z \in C$. By Proposition 7, we find a weak* open set (halfspace if one wishes) $W \subset Z^{*}$ so that $F(C) \cap W \neq \emptyset$ and $\|\cdot\|_{M_{n}}-\operatorname{diam} F(C) \cap W<2 \varepsilon_{n}$. By [7, Lemma 3.1.2], there is an open set $U \subset Z$ so that $\emptyset \neq C \cap U$ and $F(C \cap U) \subset W$. Fix some $\zeta \in F(C \cap U)$. Then

$$
\|\cdot\|_{M_{n}}-\operatorname{dist}(\partial\|\cdot\|(z), \zeta) \leq\|\cdot\|_{M_{n}}-\operatorname{dist}(F(z), \zeta)<2 \varepsilon_{n}
$$

for every $z \in C \cap U$. Thus Proposition 14 yields a Baire one mapping $f_{n}$ : $Z \rightarrow\left(Z^{*},\|\cdot\|_{M_{n}}\right)$ such that

$$
\|\cdot\|_{M_{n}}-\operatorname{dist}\left(\partial\|\cdot\|(z), f_{n}(z)\right)<2 \varepsilon_{n} \quad \text { for every } z \in Z .
$$

Further, we find continuous mappings $D_{n}^{m}: Z \rightarrow\left(B_{Z^{*}},\|\cdot\|_{M_{n}}\right), m \in \mathbb{N}$, such that $\left\|D_{n}^{m}(z)-f_{n}(z)\right\|_{M_{n}} \rightarrow 0$ as $m \rightarrow \infty$ for every $z \in Z$.

Define $M_{0}=B_{Z}$. Put

$$
\Phi\left(z^{*}\right):=\left\{\gamma \in \Gamma ;\left\langle\gamma, z^{*}\right\rangle \neq 0\right\}, \quad z^{*} \in Z^{*} ;
$$

thus $\Phi: Z^{*} \rightarrow 2^{Z}$. For $n \in \mathbb{N} \cup\{0\}$ and $m \in \mathbb{N}$ let $\|\cdot\|_{n, m}$ be the Minkowski functional of the set $M_{n}+(1 / m) B_{Z}$; this will be an equivalent norm on $Z$. For every $z \in Z$ we find a countable set $\Psi(z) \subset Z^{*}$ such that $\Psi(z) \supset$ 
$\left\{D_{n}^{m}(z) ; n, m \in \mathbb{N}\right\}$ and

$$
\|z\|_{n, m}=\sup \left\{\left\langle z, z^{*}\right\rangle ; z^{*} \in \Psi(z) \text { and }\left\|z^{*}\right\|_{n, m} \leq 1\right\}
$$

for all $n \in \mathbb{N} \cup\{0\}$ and $m \in \mathbb{N}$. Thus we have defined $\Psi: Z \rightarrow 2^{Z^{*}}$.

For the construction of the projections $P_{\alpha}: Z \rightarrow Z$ we shall use a now standard back-and-forth argument (see, e.g., [7, Section 6.1]). We need

Claim 1. Let $\aleph<\operatorname{dens} Z$ be any infinite cardinal and consider two nonempty sets $A_{0} \subset Z, B_{0} \subset Z^{*}$ with $\# A_{0} \leq \aleph, \# B_{0} \leq \aleph$. Then there exist sets $A_{0} \subset A \subset Z, B_{0} \subset B \subset Z^{*}$, closed under taking linear combinations with rational coefficients and such that $\# A \leq \aleph, \# B \leq \aleph$, and $\Phi(B) \subset A$, $\Psi(A) \subset B$.

In order to prove this, let $\operatorname{sp}_{\mathbb{Q}}$ mean the $\mathbb{Q}$-linear hull. Put $A=\bigcup_{n=1}^{\infty} A_{n}$, $B=\bigcup_{n=1}^{\infty} B_{n}$, where the sets

$A_{n}:=\operatorname{sp}_{\mathbb{Q}}\left(A_{n-1} \cup \Phi\left(B_{n-1}\right)\right), \quad B_{n}:=\operatorname{sp}_{\mathbb{Q}}\left(B_{n-1} \cup \Psi\left(A_{n}\right)\right), \quad n=1,2, \ldots$, are defined inductively. Then it is easy to verify all the proclaimed properties of the sets $A$ and $B$.

Having constructed the sets $A, B$, we observe that $\Phi(B)^{\perp} \cap \bar{B}^{*}=\{0\}$. Indeed, assume there is $0 \neq z^{*} \in \Phi(B)^{\perp} \cap \bar{B}^{*}$. Find $\gamma \in \Gamma$ so that $\left\langle\gamma, z^{*}\right\rangle \neq 0$. Find $b \in B$ so that $\langle\gamma, b\rangle \neq 0$. Then $\gamma \in \Phi(b)$. But $z^{*} \in \Phi(B)^{\perp}$ and so $\left\langle\gamma, z^{*}\right\rangle=0$, a contradiction (we have just proved that $\Phi$ is a so called projectional generator on $Z$, see [7, Section 6.1]). Therefore $A^{\perp} \cap \bar{B}^{*}=\{0\}$. [7, Lemma 6.1.1] and its proof then yield a linear projection $P: Z \rightarrow Z$ with $P Z=\bar{A}, P^{-1}(0)=B_{\perp}$, and $P^{*} Z^{*}=\bar{B}^{*}$, such that $\|P\|_{n, m}=1$ for all $n \in \mathbb{N} \cup\{0\}$ and $m \in \mathbb{N}$. Thus

$$
P M_{n} \subset \bigcap_{m=1}^{\infty} P\left(M_{n}+\frac{1}{m} B_{Z}\right) \subset \bigcap_{m=1}^{\infty} \overline{M_{n}+\frac{1}{m} B_{Z}} \subset \bigcap_{m=1}^{\infty}\left(M_{n}+\frac{2}{m} B_{Z}\right)=M_{n}
$$

for every $n \in \mathbb{N} \cup\{0\}$, and in particular, $\|P\|=1$.

Fix any $\gamma \in \Gamma$. We now prove that $P \gamma \in\{\gamma, 0\}$. If $\gamma \in P Z$, then, trivially, $P \gamma=\gamma$. Second, assume that $\gamma \notin P Z(=\bar{A})$. Then $\gamma \notin \Phi(B)$, which implies that $\langle\gamma, b\rangle=0$ for every $b \in B$, that is, $\gamma \in B_{\perp}$. But $B_{\perp}=P^{-1}(0)$. Hence $P \gamma=0$.

Now, once we know how to construct one projection $P: Z \rightarrow Z$, the construction of the whole PRI $\left(P_{\alpha} ; \omega \leq \alpha \leq \mu\right)$ is standard (see, e.g., [7, Section 6.1]). We just recall that the projections $P_{\alpha}$ "come" from sets $A_{\alpha} \subset Z$ and $B_{\alpha} \subset Z^{*}$ with several properties; in particular, they are rationally linear (that is, closed under taking linear combinations with rational coefficients) and satisfy

$$
\Phi\left(B_{\alpha}\right) \subset A_{\alpha}, \quad \Psi\left(A_{\alpha}\right) \subset B_{\alpha}, \quad \bar{A}_{\alpha}=P_{\alpha} Z, \quad \text { and } \quad \bar{B}_{\alpha}^{*}=P_{\alpha}^{*} Z^{*}
$$

for all $\omega \leq \alpha \leq \mu$, and $A_{\lambda}=\bigcup_{\beta<\lambda} A_{\beta}$ for every limit ordinal $\lambda \in(\omega, \mu]$. 
Until the end of the proof, we fix any $n \in \mathbb{N}$ and any limit ordinal $\omega<\lambda \leq \mu$. It remains to show the last assertion of our proposition. Fix for a while a subspace $Y \subset P_{\lambda} Z$. Put

$$
\Delta_{Y}:=\left[f_{n}(Y)+2 \varepsilon_{n}\left(P_{\lambda}\left(M_{n}\right) \cap Y\right)^{\circ}\right] \cap B_{P_{\lambda}^{*} Z^{*}},
$$

where $\left(P_{\lambda}\left(M_{n}\right) \cap Y\right)^{\circ}=\left\{z^{*} \in Z^{*} ; \sup \left\langle P_{\lambda}\left(M_{n}\right) \cap Y, z^{*}\right\rangle \leq 1\right\}$. This $\Delta_{Y}$ is a so called boundary for $Y$, that is, for every $z \in Y$ there exists $\delta \in \Delta_{Y}$ so that $\|z\|=\langle z, \delta\rangle$. Indeed, fix any $z \in Y$. By (2) we find $z^{*} \in \partial\|\cdot\|(z)$ so that $\left\|z^{*}-f_{n}(z)\right\|_{M_{n}}<2 \varepsilon_{n}$. Put $\delta=P_{\lambda}^{*} z^{*}$. Then $\delta \in B_{P_{\lambda}^{*} Z^{*}},\langle z, \delta\rangle=$ $\left\langle z, z^{*}\right\rangle=\|z\|$, and $\delta=f_{n}(z)+\left(\delta-f_{n}(z)\right) \in f_{n}(Y)+2 \varepsilon_{n}\left(P_{\lambda}\left(M_{n}\right) \cap Y\right)^{\circ}$, and so $\delta \in \Delta_{Y}$. Here we have used the estimate

$$
\left\|\delta-f_{n}(z)\right\|_{P_{\lambda}\left(M_{n}\right) \cap Y} \leq\left\|z^{*}-f_{n}(z)\right\|_{M_{n}}<2 \varepsilon_{n} .
$$

From now on fix any $z^{*} \in B_{Z^{*}}$. Let $C \subset A_{\lambda}$ be any countable set which is rationally linear.

Claim 2. There are $k \in \mathbb{N}$, rational numbers $a_{1}, \ldots, a_{k}$, vectors $y_{1}, \ldots, y_{k}$ $\in C$, and $m \in \mathbb{N}$ such that

$$
\sup \left\langle P_{\lambda}\left(M_{n}\right) \cap \bar{C}, P_{\lambda}^{*} z^{*}-\sum_{i=1}^{k} a_{i} D_{n}^{m}\left(y_{i}\right)\right\rangle<\frac{9}{2} \varepsilon_{n} .
$$

To prove this, put $Y=\bar{C}$; this is a subspace of $P_{\lambda} Z$. First, we show that $P_{\lambda}^{*} z^{*}$ lies in the norm closure of co $\Delta_{Y}+2 \varepsilon_{n}\left(P_{\lambda}\left(M_{n}\right) \cap Y\right)^{\circ}$. Assume this is not true. Find then $\varphi \in Z^{* *}$ and $\alpha \in \mathbb{R}$ so that

$$
\left\langle\varphi, P_{\lambda}^{*} z^{*}\right\rangle>\alpha>\sup \left\langle\varphi, \operatorname{co} \Delta_{Y}+2 \varepsilon_{n}\left(P_{\lambda}\left(M_{n}\right) \cap Y\right)^{\circ}\right\rangle .
$$

As $P_{\lambda}\left(M_{n}\right) \cap Y \subset B_{Z}$, we may and do assume that $\sup \left\langle\varphi,\left(P_{\lambda}\left(M_{n}\right) \cap Y\right)^{\circ}\right\rangle$ $=1$. Thus, by the bipolar theorem, $\varphi \in{\overline{P_{\lambda}\left(M_{n}\right) \cap Y}}^{*}$, and hence, the above inequalities have the form

$$
\left\langle\varphi, P_{\lambda}^{*} z^{*}\right\rangle>\alpha>\sup \left\langle\varphi, \Delta_{Y}\right\rangle+2 \varepsilon_{n} .
$$

Since $P_{\lambda}\left(M_{n}\right) \cap Y$ is a separable $\varepsilon_{n}$-Asplund set, there exists a countable set $S \subset \Delta_{Y}$ such that for every $\delta \in \Delta_{Y}$ there is $s \in S$ such that $\sup \left\langle P_{\lambda}\left(M_{n}\right) \cap Y\right.$, $\delta-s\rangle<\varepsilon_{n}$. As $\varphi \in{\overline{P_{\lambda}\left(M_{n}\right) \cap Y}}^{*}$, there is a sequence $\left(z_{i}\right)_{i \in \mathbb{N}}$ in $P_{\lambda}\left(M_{n}\right) \cap Y$ such that

$$
\left\langle z_{i}, P_{\lambda}^{*} z^{*}\right\rangle \rightarrow\left\langle\varphi, P_{\lambda}^{*} z^{*}\right\rangle, \quad \text { and } \quad\left\langle z_{i}, s\right\rangle \rightarrow\langle\varphi, s\rangle \quad \text { for every } s \in S
$$

as $i \rightarrow \infty$. We may and do assume that $\left\langle z_{i}, P_{\lambda}^{*} z^{*}\right\rangle>\alpha$ for all $i \in \mathbb{N}$. We now verify the assumptions of Simons' lemma (see, e.g., [7, Lemma 8.1.3]). Put $\Gamma=B_{P_{\lambda}^{*} Z^{*}}$, and for $i \in \mathbb{N}$ define $g_{i} \in \ell_{\infty}(\Gamma)$ by $g_{i}(\gamma)=\left\langle z_{i}, \gamma\right\rangle, \gamma \in \Gamma$. As $\Delta_{Y}$ is a boundary for $Y$, for any positive numbers $\lambda_{1}, \lambda_{2}, \ldots$ with $\sum_{i=1}^{\infty} \lambda_{i}=1$ there is $\gamma \in \Delta_{Y}$ so that $\left\|\sum_{i=1}^{\infty} \lambda_{i} g_{i}\right\|_{\infty}=\sum_{i=1}^{\infty} \lambda_{i} g_{i}(\gamma)$. Thus, by Simons' 
lemma, we have

$$
\sup \left\{\limsup _{i \rightarrow \infty}\left\langle z_{i}, \delta\right\rangle ; \delta \in \Delta_{Y}\right\} \geq \inf \left\{\|g\|_{\infty} ; g \in \operatorname{co}\left\{g_{i} ; i \in \mathbb{N}\right\}\right\} .
$$

But

$$
\begin{aligned}
\inf \left\{\|g\| ; g \in \operatorname{co}\left\{g_{i} ; i \in \mathbb{N}\right\}\right\} & \geq \inf \left\{\left\langle y, P_{\lambda}^{*} z^{*}\right\rangle ; y \in \operatorname{co}\left\{z_{i} ; i \in \mathbb{N}\right\}\right\} \\
& =\inf \left\{\left\langle z_{i}, P_{\lambda}^{*} z^{*}\right\rangle ; i \in \mathbb{N}\right\} \geq \alpha .
\end{aligned}
$$

Hence

$$
\sup \left\{\limsup _{i \rightarrow \infty}\left\langle z_{i}, \delta\right\rangle ; \delta \in \Delta_{Y}\right\} \geq \alpha \text {. }
$$

Now fix any $\delta \in \Delta_{Y}$. Find $s \in S$ so that $\sup \left\langle P_{\lambda}\left(M_{n}\right) \cap Y, \delta-s\right\rangle<\varepsilon_{n}$. Thus $\lim \sup \left\langle z_{i}, \delta\right\rangle$

$$
\begin{aligned}
& i \rightarrow \infty \\
& =\lim _{i \rightarrow \infty}\left\langle z_{i}, s\right\rangle+\limsup _{i \rightarrow \infty}\left\langle z_{i}, \delta-s\right\rangle\left\langle\langle\varphi, s\rangle+\varepsilon_{n} \quad\left(\text { as } z_{i} \in P_{\lambda}\left(M_{n}\right) \cap Y\right)\right. \\
& =\langle\varphi, \delta\rangle+\langle\varphi, s-\delta\rangle+\varepsilon_{n}\left\langle\langle\varphi, \delta\rangle+2 \varepsilon_{n} \quad\left(\text { as } \varphi \in{\overline{P_{\lambda}\left(M_{n}\right) \cap Y}}^{*}\right) .\right.
\end{aligned}
$$

Then (4) gives $\alpha \leq \sup \left\langle\varphi, \Delta_{Y}\right\rangle+2 \varepsilon_{n}$, contrary to (3). We have thus proved that $P_{\lambda}^{*} z^{*} \in \overline{\operatorname{co} \Delta_{Y}+2 \varepsilon_{n}\left(P_{\lambda}\left(M_{n}\right) \cap Y\right)^{\circ}}$.

Having this at hand, we find $k \in \mathbb{N}$, rational numbers $a_{1}, \ldots, a_{k}>0$ with $a_{1}+\cdots+a_{k}=1$, and $\delta_{1}, \ldots, \delta_{k} \in \Delta_{Y}$ so that

$$
\sup \left\langle P_{\lambda}\left(M_{n}\right) \cap Y, P_{\lambda}^{*} z^{*}-\sum_{i=1}^{k} a_{i} \delta_{i}\right\rangle<\frac{5}{2} \varepsilon_{n} .
$$

For $i=1, \ldots, k$ write

$$
\delta_{i}=f_{n}\left(z_{i}\right)+2 \varepsilon_{n} z_{i}^{*},
$$

where $z_{i} \in Y$ and $z_{i}^{*} \in\left(P_{\lambda}\left(M_{n}\right) \cap Y\right)^{\circ}$; this comes from the definition of $\Delta_{Y}$. Then

$$
\sup \left\langle P_{\lambda}\left(M_{n}\right) \cap Y, P_{\lambda}^{*} z^{*}-\sum_{i=1}^{k} a_{i}\left(f_{n}\left(z_{i}\right)+2 \varepsilon_{n} z_{i}^{*}\right)\right\rangle<\frac{5}{2} \varepsilon_{n}
$$

and so,

$$
\sup \left\langle P_{\lambda}\left(M_{n}\right) \cap Y, P_{\lambda}^{*} z^{*}-\sum_{i=1}^{k} a_{i} f_{n}\left(z_{i}\right)\right\rangle<\frac{9}{2} \varepsilon_{n} .
$$

Since $\left\|D_{n}^{m}\left(z_{i}\right)-f_{n}\left(z_{i}\right)\right\|_{M_{n}} \rightarrow 0$ as $m \rightarrow \infty$ for every $i=1, \ldots, k$, we can find $m \in \mathbb{N}$ so that we still have (note that $P_{\lambda}\left(M_{n}\right) \subset M_{n}$ )

$$
\sup \left\langle P_{\lambda}\left(M_{n}\right) \cap Y, P_{\lambda}^{*} z^{*}-\sum_{i=1}^{k} a_{i} D_{n}^{m}\left(z_{i}\right)\right\rangle<\frac{9}{2} \varepsilon_{n} .
$$


Since $D_{n}^{m}$ is $\|\cdot\|_{M_{n}}$-continuous and $Y=\bar{C}$, there are $y_{1}, \ldots, y_{k} \in C$ so that

$$
\sup \left\langle P_{\lambda}\left(M_{n}\right) \cap Y, P_{\lambda}^{*} z^{*}-\sum_{i=1}^{k} a_{i} D_{n}^{m}\left(y_{i}\right)\right\rangle<\frac{9}{2} \varepsilon_{n} .
$$

We have thus proved Claim 2.

It remains to perform a separable reduction in the sense that we want to show that Claim 2 holds also for $C=A_{\lambda}$, that is,

$$
\sup \left\langle P_{\lambda}\left(M_{n}\right), P_{\lambda}^{*} z^{*}-\sum_{i=1}^{k} a_{i} D_{n}^{m}\left(y_{i}\right)\right\rangle<\frac{9}{2} \varepsilon_{n}
$$

with suitable $k, m \in \mathbb{N}$, rational numbers $a_{1}, \ldots, a_{k}$, and vectors $y_{1}, \ldots, y_{k}$ $\in A_{\lambda}$. To do so, let $\mathcal{A}$ denote the set of all sequences $a=\left(a_{1}, a_{2}, \ldots\right)$ with rational entries such that $a_{i}=0$ for all large $i \in \mathbb{N}$. Note that $\mathcal{A}$ is a countable set. Pick a non-empty, at most countable set $S_{1} \subset P_{\lambda}\left(M_{n}\right)$ and find a countable rationally linear set $C_{1} \subset A_{\lambda}$ so that $\bar{C}_{1} \supset S_{1}$. Enumerate $C_{1}$ as $\left\{v_{1}^{1}, v_{2}^{1}, \ldots\right\}$. For every $m \in \mathbb{N}$ and every $a=\left(a_{i}\right) \in \mathcal{A}$ we find $z(1, m, a) \in P_{\lambda}\left(M_{n}\right)$ so that

$$
\sup \left\langle P_{\lambda}\left(M_{n}\right), P_{\lambda}^{*} z^{*}-\sum_{i=1}^{\infty} a_{i} D_{n}^{m}\left(v_{i}^{1}\right)\right\rangle-\frac{1}{1}<\left\langle z(1, m, a), P_{\lambda}^{*} z^{*}-\sum_{i=1}^{\infty} a_{i} D_{n}^{m}\left(v_{i}^{1}\right)\right\rangle .
$$

Put then $S_{2}=S_{1} \cup\{z(1, m, a) ; m \in \mathbb{N}, a \in \mathcal{A}\}$ and find a countable rationally linear set $C_{2} \subset A_{\lambda}$ such that $C_{2} \supset C_{1}$ and $\bar{C}_{2} \supset S_{2}$. Let $l \in \mathbb{N}$, and assume we have already constructed countable sets $S_{1} \subset \cdots \subset S_{l} \subset$ $P_{\lambda}\left(M_{n}\right)$ and rationally linear countable sets $C_{1} \subset \cdots \subset C_{l} \subset A_{\lambda}$ with $S_{1} \subset \bar{C}_{1}, \ldots, S_{l} \subset \bar{C}_{l}$. Enumerate $C_{l}$ as $\left\{v_{1}^{l}, v_{2}^{l}, \ldots\right\}$. For every $m \in \mathbb{N}$ and every $a \in \mathcal{A}$ we find a vector $z(l, m, a) \in P_{\lambda}\left(M_{n}\right)$ such that

$\sup \left\langle P_{\lambda}\left(M_{n}\right), P_{\lambda}^{*} z^{*}-\sum_{i=1}^{\infty} a_{i} D_{n}^{m}\left(v_{i}^{l}\right)\right\rangle-\frac{1}{l}<\left\langle z(l, m, a), P_{\lambda}^{*} z^{*}-\sum_{i=1}^{\infty} a_{i} D_{n}^{m}\left(v_{i}^{l}\right)\right\rangle$.

Put then $S_{l+1}=S_{l} \cup\{z(l, m, a) ; m \in \mathbb{N}, a \in \mathcal{A}\}$, and find a rationally linear countable set $C_{l+1} \subset A_{\lambda}$ such that $C_{l+1} \supset C_{l}$ and $\bar{C}_{l+1} \supset S_{l+1}$. Finally, having performed this for every $l \in \mathbb{N}$, put $S=\bigcup_{l=1}^{\infty} S_{l}$ and $C=\bigcup_{l=1}^{\infty} C_{l}$. Clearly, $C$ is rationally linear.

By Claim 2 , we find $k \in \mathbb{N}$, rational numbers $a_{1}, \ldots, a_{k}$, vectors $y_{1}, \ldots, y_{k}$ $\in C, m \in \mathbb{N}$, and $l \in \mathbb{N}$ such that

$$
\sup \left\langle P_{\lambda}\left(M_{n}\right) \cap \bar{C}, P_{\lambda}^{*} z^{*}-\sum_{i=1}^{k} a_{i} D_{n}^{m}\left(y_{i}\right)\right\rangle+\frac{1}{l}<\frac{9}{2} \varepsilon_{n} .
$$

By enlarging $l$ if necessary, we can achieve that $y_{1}=v_{i_{1}}^{l}, \ldots, y_{k}=v_{i_{k}}^{l}$ with suitable $i_{1}, \ldots, i_{k} \in \mathbb{N}$. Define $b=\left(b_{1}, b_{2}, \ldots\right)$ by $b_{i_{1}}=a_{1}, \ldots, b_{i_{k}}=a_{k}$, and 
$b_{i}=0$ for the remaining $i \in \mathbb{N}$. Then $b \in \mathcal{A}$ and we can estimate

$$
\begin{aligned}
\sup \left\langle P_{\lambda}\left(M_{n}\right),\right. & \left.P_{\lambda}^{*} z^{*}-\sum_{i=1}^{k} a_{i} D_{n}^{m}\left(y_{i}\right)\right\rangle \\
& =\sup \left\langle P_{\lambda}\left(M_{n}\right), P_{\lambda}^{*} z^{*}-\sum_{i=1}^{\infty} b_{i} D_{n}^{m}\left(v_{i}^{l}\right)\right\rangle \\
& <\sup \left\langle z(l, m, b), P_{\lambda}^{*} z^{*}-\sum_{i=1}^{\infty} b_{i} D_{n}^{m}\left(v_{i}^{l}\right)\right\rangle+\frac{1}{l} \\
& \leq \sup \left\langle P_{\lambda}\left(M_{n}\right) \cap \bar{C}, P_{\lambda}^{*} z^{*}-\sum_{i=1}^{k} a_{i} D_{n}^{m}\left(y_{i}\right)\right\rangle+\frac{1}{l}<\frac{9}{2} \varepsilon_{n} .
\end{aligned}
$$

This proves (5) and completes the separable reduction.

Finally, find $\beta_{0}<\lambda$ so that $y_{1}, \ldots, y_{k} \in A_{\beta_{0}}$; it exists as $A_{\lambda}=\bigcup_{\beta<\lambda} A_{\beta}$. Put then $\zeta=\sum_{i=1}^{k} a_{i} D_{n}^{m}\left(y_{i}\right)$. We observe that

$$
\zeta \in \sum_{i=1}^{k} a_{i} \Psi\left(y_{i}\right) \subset \sum_{i=1}^{k} a_{i} \Psi\left(A_{\beta_{0}}\right) \subset B_{\beta_{0}} \subset \bar{B}_{\beta_{0}}^{*}=P_{\beta_{0}}^{*} Z^{*} .
$$

Therefore for $\beta_{0}<\beta<\lambda$ we have, from (5),

$$
\begin{aligned}
\left\|P_{\lambda}^{*} z^{*}-P_{\beta}^{*} z^{*}\right\|_{M_{n}} & \leq\left\|P_{\lambda}^{*} z^{*}-\zeta\right\|_{M_{n}}+\left\|\zeta-P_{\beta}^{*} z^{*}\right\|_{M_{n}} \\
& =\sup \left\langle P_{\lambda}\left(M_{n}\right), P_{\lambda}^{*} z^{*}-\zeta\right\rangle+\sup \left\langle P_{\beta}\left(M_{n}\right), \zeta-P_{\lambda}^{*} z^{*}\right\rangle \\
& \leq 2 \sup \left\langle P_{\lambda}\left(M_{n}\right), P_{\lambda}^{*} z^{*}-\zeta\right\rangle<9 \varepsilon_{n}
\end{aligned}
$$

as $P_{\beta}\left(M_{n}\right) \subset P_{\lambda}\left(M_{n}\right)$.

We are now ready to prove (iv) $\Rightarrow$ (o) in Theorem 1 when $X$ is WLD. Note that if $X$ is WLD, then it contains a linearly dense set $\Gamma \subset B_{X}$ which countably supports all elements of $X^{*}$, that is, for every $x^{*} \in X^{*}$ the set $\left\{\gamma \in \Gamma ;\left\langle\gamma, x^{*}\right\rangle \neq 0\right\}$ is at most countable [9, Theorem 5]. In order to prove (o), by [9, Theorem 2], it suffices to show the following

Claim 1. There exists a linearly dense set $\Gamma \subset B_{X}$ such that for every $\varepsilon>0$ there are subsets $\Gamma_{i}^{\varepsilon} \subset \Gamma, i \in \mathbb{N}$, satisfying $\Gamma=\bigcup_{n=1}^{\infty} \Gamma_{n}^{\varepsilon}$, and such that for all $n \in \mathbb{N}$ and $x^{*} \in X^{*}$ the set $\left\{\gamma \in \Gamma_{n}^{\varepsilon} ;\left\langle\gamma, x^{*}\right\rangle>\varepsilon\right\}$ is finite.

Instead of proving this, we shall prove a subtler statement:

Claim 2. Let $X$ be a WLD space which is simultaneously $\sigma$-Asplund generated, with sets $A_{n}^{\varepsilon}, \varepsilon>0, n \in \mathbb{N}$, witnessing that. Let $\Gamma \subset B_{X}$ be any set which is linearly dense in $X$ and countably supports $X^{*}$. Then there exist subsets $\Gamma_{i} \subset \Gamma, i \in \mathbb{N}$, satisfying $\Gamma=\bigcup_{i=1}^{\infty} \Gamma_{i}$ and such that for every rational $\varepsilon>0$, every $n, i \in \mathbb{N}$, and every $x^{*} \in X^{*}$ the set $\{\gamma \in$ $\left.\Gamma_{i} \cap A_{n}^{\varepsilon / 9} ;\left\langle\gamma, x^{*}\right\rangle>\varepsilon\right\}$ is finite. 
Now, if for a rational $\varepsilon>0$ we put $\Gamma_{n, i}^{\varepsilon}:=\Gamma_{i} \cap A_{n}^{\varepsilon / 9}, n, i \in \mathbb{N}$, and for an irrational $\varepsilon>0$ we put $\Gamma_{n, i}^{\varepsilon}:=\Gamma_{i} \cap A_{n}^{\varepsilon^{\prime} / 9}, n, i \in \mathbb{N}$, where $\varepsilon^{\prime}$ is a fixed rational number from the interval $(\varepsilon / 2, \varepsilon)$, then, enumerating $\mathbb{N} \times \mathbb{N}$ by elements of $\mathbb{N}$, we get Claim 1 .

Claim 2 will be proved by transfinite induction on the density of $X$. The case when $X$ is separable is simple. Further, let $\aleph$ be an uncountable cardinal, and assume that Claim 2 has already been proved for all spaces $X$ with density less than $\aleph$. Now let $X$ be a Banach space with density and satisfying the assumptions of Claim 2. Let $\Gamma \subset B_{X}$ be any set which is linearly dense in $X$ and countably supports $X^{*}$. We replace each set $A_{n}^{\varepsilon}$ by its absolutely convex closed hull; this change will not affect anything. Applying Proposition 15, we get a PRI $\left(P_{\alpha} ; \omega \leq \alpha \leq \mu\right)$ on $X$ such that $P_{\alpha}\left(A_{n}^{\varepsilon / 9}\right) \subset A_{n}^{\varepsilon / 9}, P_{\alpha}(\gamma) \in\{\gamma, 0\}$ for every $\alpha \in[\omega, \mu]$, every $n \in \mathbb{N}$, every rational $\varepsilon>0$, and every $\gamma \in \Gamma$; and moreover, for every limit ordinal $\omega<\lambda \leq \mu$, every $n \in \mathbb{N}$, every $\varepsilon>0$, and every $x^{*} \in B_{X^{*}}$ we have

$$
\limsup _{\beta \uparrow \lambda} \sup \left\langle A_{n}^{\varepsilon / 9}, P_{\lambda}^{*} x^{*}-P_{\beta}^{*} x^{*}\right\rangle<\varepsilon .
$$

Fix any $\omega \leq \alpha<\mu$ and write $Q_{\alpha}=P_{\alpha+1}-P_{\alpha}$. Then the (complemented) subspace $Q_{\alpha} X$ is also WLD, $B_{Q_{\alpha} X}=\bigcup_{n=1}^{\infty}\left(Q_{\alpha} X \cap A_{n}^{\varepsilon}\right)$ for every $\varepsilon>0$, and each set $Q_{\alpha} X \cap A_{n}^{\varepsilon}$ is $\varepsilon$-Asplund in $Q_{\alpha} X$. Also $Q_{\alpha} \Gamma$ is linearly dense in $Q_{\alpha} X, Q_{\alpha} \Gamma \subset \Gamma$, and $Q_{\alpha} \Gamma$ countably supports the dual $\left(Q_{\alpha} X\right)^{*}$. Thus the assumptions of Claim 2 are satisfied for the subspace $Q_{\alpha} X$. Then, by the induction assumption, there are $\Gamma_{i}^{\alpha} \subset Q_{\alpha} \Gamma, i \in \mathbb{N}$, satisfying $Q_{\alpha} \Gamma=$ $\bigcup_{i=1}^{\infty} \Gamma_{i}^{\alpha}$ and such that for every rational $\varepsilon>0$,

$$
\forall n, i \in \mathbb{N} \forall y^{*} \in\left(Q_{\alpha} X\right)^{*} \quad \#\left\{\gamma \in \Gamma_{i}^{\alpha} \cap\left(Q_{\alpha} X \cap A_{n}^{\varepsilon / 9}\right) ;\left\langle\gamma, y^{*}\right\rangle>\varepsilon\right\}<\omega .
$$

Assume that we have done the above for every $\omega \leq \alpha<\mu$. Put then $\Gamma_{i}=$ $\bigcup_{\omega \leq \alpha<\mu} \Gamma_{i}^{\alpha}, i \in \mathbb{N}$. Clearly $\Gamma=\bigcup_{i=1}^{\infty} \Gamma_{i}$.

Now fix any rational $\varepsilon>0, n, i \in \mathbb{N}$, and $x^{*} \in X^{*}$. Define

$$
\Lambda=\left\{\alpha \in[\omega, \mu) ;\left\langle\gamma, x^{*}\right\rangle>\varepsilon \text { for some } \gamma \in \Gamma_{i}^{\alpha} \cap A_{n}^{\varepsilon / 9}\right\} .
$$

We observe that

$$
\left\{\gamma \in \Gamma_{i} \cap A_{n}^{\varepsilon / 9} ;\left\langle\gamma, x^{*}\right\rangle>\varepsilon\right\}=\bigcup_{\alpha \in \Lambda}\left\{\gamma \in \Gamma_{i}^{\alpha} \cap A_{n}^{\varepsilon / 9} ;\left\langle\gamma, x_{\mid Q_{\alpha} X}^{*}\right\rangle>\varepsilon\right\}
$$

and that each set from this union is finite, by the induction assumption. Hence it remains to show that $\Lambda$ is finite.

Assume not. Then there exists an infinite injective sequence $\gamma_{1}, \gamma_{2}, \ldots$ in $\Gamma_{i} \cap A_{n}^{\varepsilon / 9}$ such that $\left\langle\gamma_{j}, x^{*}\right\rangle>\varepsilon$ for every $j \in \mathbb{N}$. For each $j \in \mathbb{N}$ we find $\alpha_{j}<\mu$ so that $\gamma_{j} \in \Gamma_{i}^{\alpha_{j}}\left(\subset Q_{\alpha_{j}} X\right)$. By suppressing some $\alpha_{j}$ 's and reindexing, we can achieve that $\alpha_{1}<\alpha_{2}<\cdots$. Put $\lambda=\lim _{j \rightarrow \infty} \alpha_{j}$; then $\lambda \leq \mu$. From (6) we find $j \in \mathbb{N}$ such that $\sup \left\langle A_{n}^{\varepsilon / 9}, P_{\lambda}^{*} x^{*}-P_{\alpha_{l}}^{*} x^{*}\right\rangle<\varepsilon$ 
whenever $l \in \mathbb{N}$ and $l \geq j$. Then we have

$$
\begin{aligned}
\varepsilon & <\left\langle\gamma_{j+1}, x^{*}\right\rangle=\left\langle P_{\lambda} \gamma_{j+1}, x^{*}\right\rangle \\
& =\left\langle\gamma_{j+1}, P_{\lambda}^{*} x^{*}-P_{\alpha_{j}}^{*} x^{*}\right\rangle+\left\langle P_{\alpha_{j}} \gamma_{j+1}, x^{*}\right\rangle<\varepsilon+0,
\end{aligned}
$$

a contradiction. Therefore the set $\Lambda$ must be finite.

Claim 2 is thus proved and the proof of Theorem 1 is finished.

REMARK. From Proposition 15 we can also deduce a well known result that a Banach space $X$ is WCG if (and only if) it is simultaneously WLD and Asplund generated (see, e.g., [7, Theorem 8.3.4]). Indeed, let $M$ be a linearly dense convex symmetric closed Asplund set in a WLD space $X$. Put $\varepsilon_{n}=1 / n$ and $M_{n}=M, n \in \mathbb{N}$. Then Proposition 15 yields a $\operatorname{PRI}\left(P_{\alpha}\right)$ on $X$ so that $P_{\alpha} M \subset M$ for all $\alpha$, and for every limit ordinal $\lambda$ and every $x^{*} \in X^{*}$ we have $\left\|P_{\lambda}^{*} x^{*}-P_{\beta}^{*} x^{*}\right\|_{M} \rightarrow 0$ as $\beta \uparrow \lambda$. Now a standard argument shows that $X$ is WCG (see, e.g., [14]).

Proof of Theorem 3. (i) $\Rightarrow$ (ii). Assume that the compact space $K$ is Eberlein. The space $C(K)$ is then WCG (see [1]), and Theorem 1 implies that $C(K)$ is $\sigma$-Asplund generated. That $K$ is a Corson compact follows for instance from [7, Theorem 7.2.7].

The equivalence (ii) $\Leftrightarrow$ (iii) follows from Avilés' result [3, Theorem 20].

(ii) $\Rightarrow(\mathrm{i})$. In the proof of $[7$, Theorem 8.3.5] we can find the following

Claim. Let $\varepsilon>0$, let $\mu$ be a probability measure on $K$, let $M \subset K$ be a Borel set with $\mu(M)>0$, and let $A \subset B_{C(K)}$ be an $\varepsilon$-Asplund set. Then there exists a closed set $L \subset M$ such that $\mu(L)>0$ and $A$-diam $L<\varepsilon$.

Let $A_{n}^{\varepsilon} \subset B_{C(K)}, \varepsilon>0, n \in \mathbb{N}$, be the sets witnessing that $C(K)$ is $\sigma$-Asplund generated. Fix any $n, m \in \mathbb{N}$. Let $\mathcal{F}_{n}^{m}$ be a maximal family of mutually disjoint closed subsets $L \subset K$ such that $\mu(L)>0$ and $A_{n}^{1 / m}$-diam $L<1 / m$. Put $H_{n}^{m}=\bigcup \mathcal{F}_{n}^{m}$; this is an $F_{\sigma}$, hence Borel set. We now show that $\mu\left(H_{n}^{m}\right)=1$. Assume not. Then $\mu\left(K \backslash H_{n}^{m}\right)>0$. By the above claim, we find a closed set $L \subset K \backslash H_{n}^{m}$ such that $\mu(L)>0$ and $A_{n}^{1 / m}$-diam $L<1 / m$. But this contradicts the maximality of the family $\mathcal{F}_{n}^{m}$. Therefore $\mu\left(H_{n}^{m}\right)=1$. Clearly, the family $\mathcal{F}_{n}^{m}$ is at most countable. Find an at most countable subset $S_{n}^{m} \subset H_{n}^{m}$ such that for every $k \in H_{n}^{m}$ there is $s \in S_{n}^{m}$ such that $\sup \left\{|f(k)-f(s)| ; f \in A_{n}^{1 / m}\right\}<1 / m$.

Having done the above for all $n, m \in \mathbb{N}$, put

$$
H=\bigcap_{n, m=1}^{\infty} H_{n}^{m} \text { and } S=\bigcup_{n, m=1}^{\infty} S_{n}^{m} .
$$

Note that $H$ is a Borel set, $\mu(H)=1$, and $S$ is at most countable. We now show that $H \subset \bar{S}$. Let $f \in B_{C(K)}$ be any function. It is enough to show that $f(H) \subset \overline{f(S)}$. So fix any $k \in H$ and any $\varepsilon>0$. Find $m \in \mathbb{N}$ so that $1 / m<\varepsilon$. 
Find then $n \in \mathbb{N}$ so that $f \in A_{n}^{1 / m}$. Since $k \in H_{n}^{m}$, there is an $s \in S_{n}^{m}(\subset S)$ such that $|f(k)-f(s)|<\varepsilon$. We have proved that $f(H) \subset \overline{f(S)}$ for every $f \in B_{C(K)}$, and therefore $H \subset \bar{S}$.

Now, since $K$ is a Corson compact space, we may and do assume that $K \subset\left(\Sigma(\Gamma)\right.$, pointwise) for a suitable set $\Gamma$. Find a countable set $\Gamma_{0} \subset \Gamma$ such that $s(\gamma)=0$ whenever $s \in S$ and $\gamma \in \Gamma \backslash \Gamma_{0}$. Then also $k(\gamma)=0$ whenever $k \in H$ and $\gamma \in \Gamma \backslash \Gamma_{0}$. It follows that $H$ is a separable subset of $K$. Recalling that $\mu(H)=1$, we can conclude that $\mu$ has a separable support. Then, by, e.g., [7, Lemma 7.3.5], the Banach space $C(K)$ is WLD.

Finally, once we know that $C(K)$ is both WLD and $\sigma$-Asplund generated, Theorem 1 guarantees that $C(K)$ is a subspace of a WCG space. Then $[9$, Theorem 2] shows that the ball $\left(B_{C(K)^{*}}, w^{*}\right)$ is an Eberlein compact space, and hence, a fortiori, so is $K$.

REMARK. We have recently proved that (ii) $\Rightarrow$ (i) in Theorem 1 . The proof, mostly based on ideas of M. Raja [26, 27], is longer and completely different from the methods used in this paper. Therefore we have decided to publish it elsewhere.

Acknowledgements. We would like to thank the referees for their comments and suggestions that improved the final version of this paper.

Research of M. Fabian was supported by grants A 1019301, IAA 100190 610, and Institutional Research Plan of the Academy of Sciences of Czech Republic No. AVOZ 101905 03. He thanks the Department of Mathematics of the Polytechnical University of Valencia for their support and hospitality.

Research of V. Montesinos was supported in part by Project BFM200201423, the Universidad Politécnica de Valencia (Spain) and the Generalitat Valenciana. He thanks the Department of Mathematics of the University of Alberta in Edmonton, and the Institute of Mathematics of the Academy of Sciences of Czech Republic for their support and hospitality.

Research of V. Zizler was supported by grants NSERC 7926 (Canada), and IAA 100190 502, and Institutional Research Plan of the Academy of Sciences of Czech Republic No. AVOZ 10190503.

\section{References}

[1] D. Amir and J. Lindenstrauss, The structure of weakly compact sets in Banach spaces, Ann. of Math. 88 (1968), 35-46.

[2] A. D. Arvanitakis, Some remarks on Radon-Nikodým compact spaces, Studia Math. 172 (2002), 41-60.

[3] A. Avilés, Radon-Nikodým compact spaces of low weight and Banach spaces, ibid. 166 (2005), 71-82.

[4] R. Deville, G. Godefroy and V. Zizler, Smoothness and Renormings in Banach Spaces, Pitman Monogr. 64, Longman, London, 1993. 
[5] D. van Dulst and I. Namioka, A note on trees in conjugate Banach spaces, Indag. Math. 87 (1984), 7-10.

[6] R. Engelking, General Topology, PWN, Warszawa, 1977.

[7] M. Fabian, Gâteaux Differentiability of Convex Functions and Topology. Weak Asplund Spaces, Wiley, New York, 1997.

[8] -, Overclasses of the class of Radon-Nikodým compact spaces, in: Methods in Banach Spaces, J. M. F. Castillo and W. B. Johnson (eds.), London Math. Soc. Lecture Note Ser. 337, Cambridge Univ. Press, 2006, 197-214.

[9] M. Fabian, G. Godefroy, V. Montesinos and V. Zizler, Inner characterizations of weakly compactly generated Banach spaces and their relatives, J. Math. Anal. Appl. 297 (2004), 419-455.

[10] M. Fabian, G. Godefroy and V. Zizler, The structure of uniformly Gâteaux smooth Banach spaces, Israel Math. J., 124 (2001), 243-252.

[11] M. Fabian, P. Habala, P. Hájek, J. Pelant, V. Montesinos and V. Zizler, Functional Analysis and Infinite Dimensional Geometry, Canad. Math. Soc. Books Math. 8, Springer, New York, 2001.

[12] M. Fabian, P. Hájek, V. Montesinos and V. Zizler, A quantitative version of Krein's theorem, Rev. Mat. Iberoamericana 21 (2005), 237-248.

[13] M. Fabian, M. Heisler and E. Matoušková, Remarks on continuous images of RadonNikodým compacta, Comment. Math. Univ. Carolin. 39 (1998), 59-69.

[14] M. Fabian, V. Montesinos and V. Zizler, Weakly compact sets and smooth norms in Banach spaces, Bull. Austral. Math. Soc. 65 (2002), 223-230.

[15] - , - - - A characterization of subspaces of weakly compactly generated spaces, J. London Math. Soc. 69 (2004), 457-464.

[16] M. Fabian and J. H. M. Whitfield, On equivalent characterization of weakly compactly generated Banach spaces, Rocky Mountain J. Math. 24 (1994), 1363-1378.

[17] A. S. Granero, P. Hájek, and V. Montesinos, Convexity and $w^{*}$-compactness in Banach spaces, Math. Ann. 328 (2004), 625-631.

[18] J. E. Jayne, J. Orihuela, A. J. Palarés and G. Vera, $\sigma$-fragmentability of multivalued mappings and selection theorems, J. Funct. Anal. 117 (1993), 243-273.

[19] K. John and V. Zizler, Smoothness and its equivalents in weakly compactly generated Banach spaces, ibid. 15 (1974), 161-166.

[20] V. L. Klee, Some new results on smoothness and rotundity in normed linear spaces, Math. Ann. 139 (1959), 51-63.

[21] S. Mercourakis and S. Negrepontis, Banach spaces and topology II, in: Recent Progress in General Topology, M. Hušek and J. van Mill (eds.), 1992, 493-536.

[22] M. Muñoz Guillermo, Índice de K-determinación de espacios topológicos y $\sigma$-fragmentabilidad de aplicaciones, PhD Thesis, Univ. de Murcia, 2004.

[23] I. Namioka, Radon-Nikodým compact spaces and fragmentability, Mathematika 34 (1987), 258-281.

[24] I. Namioka and R. R. Phelps, Banach spaces which are Asplund spaces, Duke Math. J. 42 (1975), 735-750.

[25] R. R. Phelps, Convex Functions, Monotone Operators and Differentiability, Lecture Notes in Math. 1364, Springer, Berlin, 1993.

[26] M. Raja, On locally uniformly rotund norms, Mathematika 46 (1999), 343-358.

$[27]-$, Weak $k^{*}$ locally uniformly rotund norms and descriptive compact spaces, J. Funct. Anal. 197 (2003), 1-13.

[28] H. P. Rosenthal, The heredity problem for weakly compactly generated Banach spaces, Compos. Math. 28 (1974), 83-111. 
[29] X. Y. Zheng, Measure of non-Radon-Nikodým property and differentiability of convex functions on Banach spaces, Set-Valued Anal. 131 (2005), 181-196.

[30] V. Zizler, Nonseparable Banach spaces, in: Handbook of the Geometry of Banach Spaces, W. B. Johnson and J. Lindenstrauss (eds.), Vol. 2, North-Holland, 2003, 1743-1816.

Mathematical Institute

Czech Academy of Sciences

Žitná 25

11567 Praha 1, Czech Republic

E-mail: fabian@math.cas.cz

zizler@math.cas.cz
Departamento de Matemática Aplicada

ETSI Telecomunicación

Universidad Politécnica de Valencia

$\mathrm{C} /$ Vera, $\mathrm{s} / \mathrm{n}$

46071 Valencia, Spain

E-mail: vmontesinos@mat.upv.es

Received December 8, 2005

Revised version March 6, 2007 